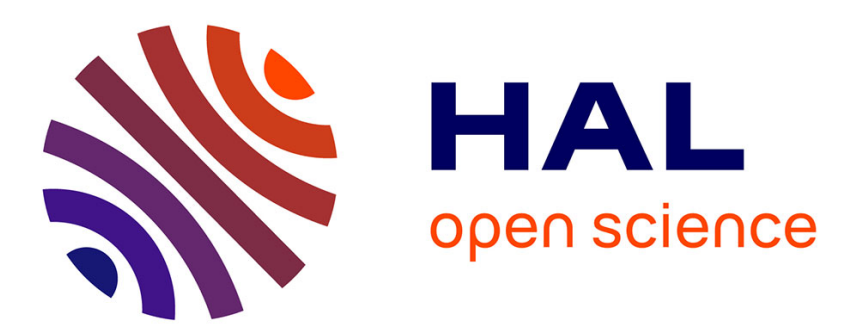

\title{
Influence of the size and skin thickness of apple varieties on the retrieval of internal optical properties using Vis/NIR spectroscopy: A Monte Carlo-based study
}

Fabrice Vaudelle, Jean-Pierre L'Huillier

\section{- To cite this version:}

Fabrice Vaudelle, Jean-Pierre L'Huillier. Influence of the size and skin thickness of apple varieties on the retrieval of internal optical properties using Vis/NIR spectroscopy: A Monte Carlo-based study. Computers and Electronics in Agriculture, 2015, 116, pp.137-149. 10.1016/j.compag.2015.06.009 . hal-02486150

\author{
HAL Id: hal-02486150 \\ https://hal.science/hal-02486150
}

Submitted on 20 Feb 2020

HAL is a multi-disciplinary open access archive for the deposit and dissemination of scientific research documents, whether they are published or not. The documents may come from teaching and research institutions in France or abroad, or from public or private research centers.
L'archive ouverte pluridisciplinaire HAL, est destinée au dépôt et à la diffusion de documents scientifiques de niveau recherche, publiés ou non, émanant des établissements d'enseignement et de recherche français ou étrangers, des laboratoires publics ou privés. 


\title{
Influence of the size and skin thickness of apple varieties on the retrieval of internal optical properties using Vis/NIR spectroscopy: A Monte Carlo-based study
}

\author{
Fabrice Vaudelle*, Jean-Pierre L'Huillier \\ PRES LUNAM, Arts-et-Métiers ParisTech, LAMPA, 2 Boulevard du Ronceray, 49035 Angers Cedex, France
}

Keywords:

Apple

Size and skin

Flesh optical properties

Inversion procedure

Vis/NIR spectroscopy

Monte Carlo simulation

\begin{abstract}
A B S T R A C T
Visible/near-infrared spectroscopy is a well-established method to measure optical properties of tissues, assuming that a light propagation model can be used to recover absorption and reduced scattering coefficients from non-invasive probing. Spectroscopic measurements have achieved success in non-destructive assessment of apple optical properties and quality attributes. However, the spectroscopy of apples must consider the size of the fruit and the presence of the thin skin layer that surrounds the flesh, to correctly read the signals acquired on the boundary. In this research, the fruit was modelled as a two layer spherical structure with various radii and finite thickness of the upper skin layer. Monte Carlo computations were performed to generate time-resolved reflectance and spatially-resolved reflectance measurements. Simulated data were then fitted using a procedure based on Levenberg-Marquardt algorithm with specific semi-infinite models. The errors in the retrieved optical properties of the flesh (absorption coefficient $\mu_{a}$, and reduced scattering coefficient $\mu_{s}^{\prime}$ ) were studied as functions of apple radius, skin thickness, and source-detector distance, for given optical parameter sets assigned to the flesh and the skin. The results suggest that the time-resolved reflectance spectroscopy may probe optical properties of the flesh regardless of the skin layer, when a sufficient source-detector distance $(15 \mathrm{~mm})$ is used for the measurements. Similar results were found in case of using the spatially resolved spectroscopy, because measurements extend up to $15-29 \mathrm{~mm}$ by steps of $1 \mathrm{~mm}$ or $2 \mathrm{~mm}$. The computations also show that the curvature of the boundary has noticeable effect on the errors in the retrieved optical coefficients of the flesh. However, results from time-resolved spectroscopy are more influenced by the size of apples, compared with the spatially-resolved spectroscopy.
\end{abstract}

\section{Introduction}

Understanding the fundamental light-tissue interaction processes is an important step towards the development of medical imaging, laser therapy, and systems to measure features of a variety of fruits. Over the past decade, a great interest was centred onto the optical characterization of agricultural and food products, by way of non-destructive tests (Nicolai et al., 2007; Zude, 2008). Conceptually, when a collimated light beam strikes a biological tissue, a fraction of the radiation is reflected at the boundary, whereas the remaining part penetrates the target. Multiple scattering and absorption act for beam broadening and dispersion, thus contributing to determine the overall light attenuation. The feature physical

\footnotetext{
* Corresponding author.

E-mail addresses: f.vaudelle@libertysurf.fr (F. Vaudelle), jean-pierre.lhuillier@ angers.ensam.fr (J.-P. L'Huillier).
}

parameters related to these events are the scattering coefficient $\mu_{s}$ (the number of scattering events per unit length), the absorption coefficient $\mu_{a}$ (the number of absorption events per unit length), and the relative refractive index $n_{r}$ (Tuchin, 2007). Due to anisotropy in light propagation, the reduced scattering coefficient $\mu_{s}^{\prime}=\mu_{s}(1-g)$ is often used and defined in terms of $\mu_{s}$ and of the angular dependence of scattering, where $g$ is the average cosine of the scattering angle. In the case of multiple scattering, the incident light intensity decreases exponentially according to the effective attenuation coefficient $\mu_{\text {eff }}=\sqrt{3 \mu_{a}\left(\mu_{a}+\mu_{s}^{\prime}\right)}$, whose reciprocal $1 / \mu_{\text {eff }}$ represents the light penetration depth at $1 / e$ ( $\sim 37 \%$ ) (Wilson and Jacques, 1990). Light scattering is primarily due to structural changes which occur in the turbid material (membranes, vacuoles, particle size, interfaces between cells). Light absorption is mainly determined by chromophores and chemical constituents (chlorophyll, sugar, water, haemoglobin). Owing to a similar spectral feature trend in the visible and 
near-infrared range (Taroni et al., 2003), fruits and human tissues may be investigated using the basic concepts which refer to the tissue optics (Welch and van Gemert, 1995). In this field of research, the first goal is to develop efficient methods by which absorbing and scattering properties of tissues can be retrieved. The second goal is to compute light propagation by means of analytical or numerical models using the optical properties as input parameters. Optical spectroscopy methods are concerned with two classes of radiation regimes which require to making use of very different types of light source and detector (see Gibson et al. 2005). The former (spatially-resolved spectroscopy) uses a continuous source of illumination, and consists in recording the re-emitted light intensity (by optical fibre setup or CCD camera) at various source-detector distances, on the tissue boundary. The measured reflectance profile may be then fitted by an appropriate mathematical framework to extract the optical parameters $\mu_{a}$ and $\mu_{s}^{\prime}$ of the interrogated tissue. The second (time-resolved spectroscopy) measures the temporal broadening of an initial short light pulse as it interacts with the tissue sample. The broadened pulse shape recorded at a specified distance from the source impact (by photon-counting detectors, i.e., time-correlated single photon counting - TCSPC) contains quantitative information related to times of flight and intensity levels. Thus, measurements of the time-dependent propagation characteristics of short light pulses in tissues present the major feature to separate the contributions of absorption from scattering. This allows to precisely assess the tissue optical properties $\left(\mu_{a}\right.$ and $\left.\mu_{s}^{\prime}\right)$, provided that an appropriate diffusion model is used in a fitting procedure.

Apple species have been the subject of experimental and theoretical studies based on light transport process. Cubbedu et al. (2001) reported on the use of time-resolved spectroscopy to assess the optical properties of apples and fruits in the wavelength 650$1000 \mathrm{~nm}$. Qin and Lu (2008) developed a novel spatially-resolved hyper spectral diffuse reflectance imaging for measuring the optical properties of fruits including apples and vegetables. They showed the interest to take into account the (approximated) spherical shape of the apples and then corrected the data acquired by a camera on the basis of acceptance angle of the optical system. Saeys et al. (2008) studied the optical properties of apple skin and flesh samples in the wavelength range from 350 to $2200 \mathrm{~nm}$, using single integrating sphere combined with inverse adding-doubling technique (Prahl, 1995). They emphasized the role played by the skin, and then suggested to model the apple as at least a two-layer structure comprising the skin and the flesh. Other works based on laser light backscattering image analysis (Park et al., 2003; Lu, 2004; Lu and Peng, 2007; Peng and Lu, 2008) have been also developed for predicting firmness and soluble solid content in apple varieties. More recently, Nguyen DoTrong et al. (2014) evaluated an original non-destructive concept based on a metamodelling approach in combination with short source-detector distances (0.3-1.2 mm), for apple quality assessment.

Besides these experiments, which often require the use of the diffusion approximation model (in direct or inverse mode), mathematical methods have been applied to numerically model the complex light-tissue interaction. Finite element (Arridge et al., 1993; Aydin et al., 2005; Deulin and L'Huillier, 2006), Boundary element (Fedele et al., 2005) and discrete ordinates (Tuchin, 1997) methods have successfully been used to model light transport in various turbid media. Monte Carlo method previously reported by Wang et al. (1995) has been often adapted to predict light propagation in multilayered tissues such as human head (Okada et al., 1997; Boas et al., 2002; Mansouri et al., 2010; Vaudelle and L'Huillier, 2013), skin (Verkruysse et al., 1993), aorta (Keijzer et al., 1989) and in other tissue arrangements (Zolek et al., 2006; Guo et al., 2008). The Monte Carlo method seems also to be especially promising in the context of food and agricultural products. Fraser et al. (2003) modeled a mandarin fruit as homogeneous sphere $(50 \mathrm{~mm})$ of tissue surrounded by an infinitesimally thin reflective boundary. They showed that Monte Carlo (MC) simulations can approximate experimental light levels in the flesh, along selected axial directions. Qin and Lu (2009) performed simulations by using a MC code and reported the major features of light transport in apples. Baranyai and Zude (2009) investigated the laser light propagation inside kiwi fruit by means of backscattering imaging and modeling based on Monte Carlo method. Wang and $\mathrm{Li}$ (2013) provided fundamental understanding of optical properties of onion tissues and light propagation in onion bulbs at $633 \mathrm{~nm}$, by performing measurements and Monte Carlo simulations.

In fact, the boundary curvature is critical for modelling the light propagation because the penetration depth $1 / \mu_{\text {eff }}$ will be significant compared with the fruit size. According to Wilson and Jacques (1990), the fruit surface cannot be viewed as a plane air-tissue interface (semi-infinite model) if the model radius is less than $12 / \mu_{\text {eff. }}$ This simplification would require very large size fruit with radii greater than $219 \mathrm{~mm}$ for $\mu_{a}=0.001 \mathrm{~mm}^{-1}$ and $\mu_{s}^{\prime}=1 \mathrm{~mm}^{-1}$, or $68 \mathrm{~mm}$ for $\mu_{a}=0.01 \mathrm{~mm}^{-1}$ and $\mu_{s}^{\prime}=1 \mathrm{~mm}^{-1}$. These radii are largely greater than the mean known values of $32 \mathrm{~mm}$ (Keshavayzpour and Rashidi, 2011) or $40 \mathrm{~mm}$ (Qin and Lu, 2008) for practically encountered apple varieties. Furthermore, the individual components such as skin and flesh, which have distinct optical properties, may be also considered to model at best the apple structure.

In this paper, Monte Carlo simulations are performed on apples assimilated to a spherical shape. Computational results are presented for cases of time-resolved reflectance and spatially-resolved tissue-arrangements, using different sourcedetector configurations. Time-dependent and steady-state signals were generated from the consideration of geometric parameters and optical properties (flesh and skin) as reported in the literature for different apple species.

Specific objectives of this research were to:

- Develop a Monte Carlo code and test its usefulness using a systematic comparison between generated signals and those derived from analytical solutions in semi-infinite and spherical configurations.

- Examine the effects of apple size and skin features on the assessment of flesh optical properties, using inversion procedures based on mismatched diffusion models of a semi-infinite homogeneous medium.

- Give information on source-detector distances that provide best internal estimates, taking into account the skin layer overlying the flesh.

\section{Material and methods}

\subsection{Monte Carlo model}

Monte Carlo (MC) simulations were performed to model the light propagation inside and at the boundary of a two-layer apple tissue. At this stage, the apple is modelled as two centred homogeneous spheres whose difference between the radii is equal to the (thin) skin thickness, whereas the inner sphere contains the flesh (Fig. 1(a) and (b)). The code provided by Wang et al. (1995) was essentially modified to simulate the time-domain reflectance spectroscopy (Vaudelle and L'Huillier, 2013), where a very short light pulse ( $\sim 4-5 \mathrm{ps})$ enters at one point at the edge of the spherical model, experiences complicated paths inside the two-layer turbid model, before reaching the detector located at some distance from the light entry point. The detector will then measure photon 
(a)

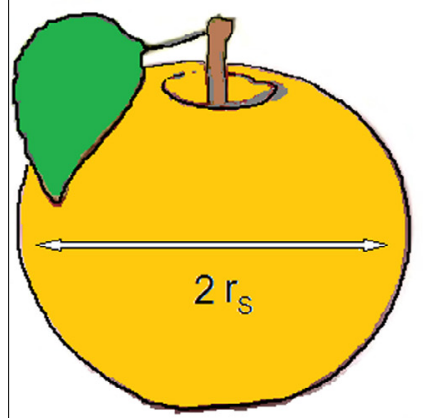

(b)

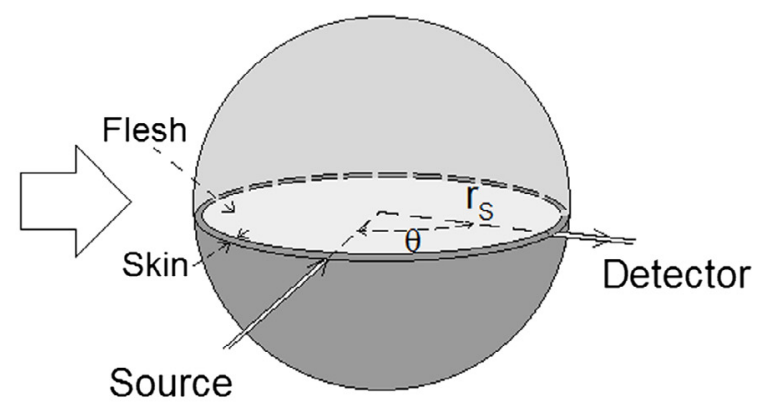

(c)

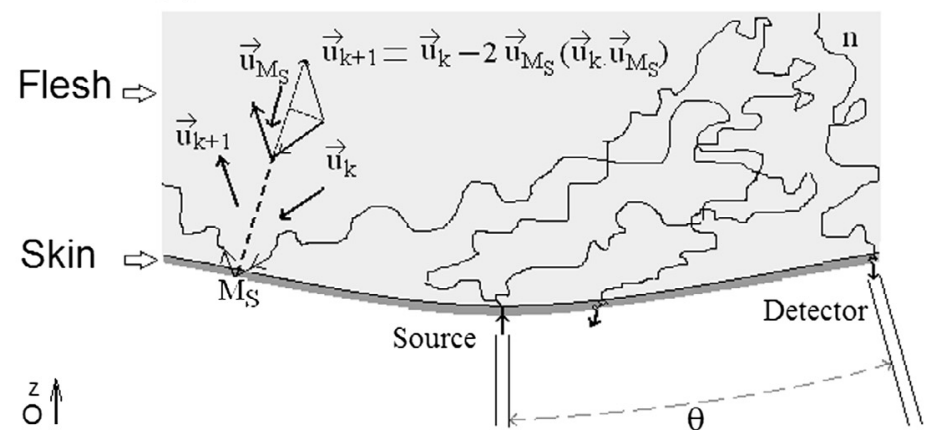

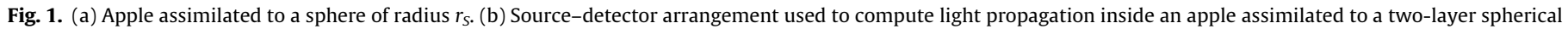

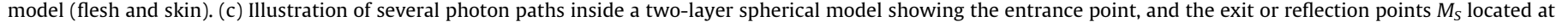
the boundary.

time-of-flight distributions which are comparable to those recorded in TCSPC operations.

Photons (packets of photons) were injected into the turbid medium from a point along the $z$ direction (Fig. 1(c)) and their paths were followed, taking into account absorption, diffusion and refraction rules, until they were scored at the detector. According to the reference code described by Wang et al. (1995), these different events occur during the application of random numbers $X_{i}$, in the interval $[0,1]$. In brief, a weight $W$ is assigned to each group of photons, with a starting value $W_{0}=1$. The step size, $S$, between successive interactions is computed as $S=-\ln \left(X_{1}\right) /\left(\mu_{a}+\mu_{s}\right)$, where the mean free path $1 /\left(\mu_{a}+\mu_{s}\right)$ represents the mean distance between absorption or scattering events. At each interaction point, the weight $\mathrm{W}_{\mathrm{k}}$ of a group of photons is reduced by the fraction $\mu_{a} /\left(\mu_{a}+\mu_{s}\right)$, so that $W_{k}=\left[1-\mu_{a} /\left(\mu_{a}+\mu_{s}\right)\right] W_{k-1}$, until the criterion $\left[W_{k}-W_{\text {threshold }}\right]<0$ is verified (a technique called as "Russian roulette" is then used to update the weight of the group of photons). Once the group of photons has been moved and its weight $\mathrm{W}_{\mathrm{k}}$ decremented, the photons are ready to be scattered. A test based on the comparison between the cosine direction $\overrightarrow{u_{r}}$ and the previous $\overrightarrow{u_{r}}$ through the relation $\left\|\overrightarrow{u_{r}}-g \overrightarrow{u_{r}^{\prime}}\right\|=\left(1-g^{2}\right) /\left(1-g+2 g X_{2}\right)$ (derived from the HenyeyGreenstein model) is used to predict the scattering scenario.

Snell's law with Fresnel's law are also considered to decide whether or not photons are reflected (transmitted) by the airtissue boundary or transmitted from the first region (skin) into the second layer of interest (flesh). These approaches require more formulae in case of spherical surface than for a plane surface. Then, the location of the contact point $\mathrm{M}_{\mathrm{S}}$ with the sphere may be determined, whereas the calculation of the coefficient of reflection $R_{M_{S}}\left(M_{S}, \overrightarrow{u_{r}}, \widehat{\overrightarrow{u_{M_{S}}}}\right)$ allows to test the refraction. The change of direction occurring in case of reflection from $M_{S}$ involves that $\overrightarrow{u_{r}} \rightarrow \overrightarrow{u_{r}}-2 \overrightarrow{u_{M_{s}}}\left(\overrightarrow{u_{M_{s}}} \cdot \overrightarrow{u_{r}}\right)$.
The Monte Carlo simulations require to use the optical parameters, which are assigned to each bulk layer composing the apple model. For convenience, the absorption and reduced scattering coefficients which are related to the skin and flesh are respectively noted as $\mu_{a}^{(\mathrm{S})}-\mu_{s}^{\prime(\mathrm{S})}$ and $\mu_{a}^{(\mathrm{f})}-\mu_{s}^{\prime}{ }^{(\mathrm{f})}$. On the other hand, the photon source may be adapted to the structure of the apple model. If the apple is reduced to an homogeneous spherical geometry (flesh), then the photons are launched at the depth $1 / \mu_{s}^{\prime}$ (f), according to an isotropic point source. When the two-layer spherical model (skin and flesh) is considered, a random isotropic source acting at the skin surface is used, with a radius equal to the mean free path of the flesh $1 /\left(\mu_{a}^{(\mathrm{f})}+\mu_{s}{ }^{(\mathrm{f})}\right)$. This allows to overcome the difficulty in modelling the photons entrance through a very thin skin layer (thickness $<1 / \mu_{s}^{\prime(\mathrm{S})}$ ).

The computed data also depend on the time $t_{i}$ spent by escaping photons and on the definition of the source-detector distance. The time $t_{i}$ is calculated as $t_{i}=n_{r} L_{i} / c$, where $L_{i}$ is the total path-length of the photons, $c$ the velocity of light in the vacuum, and $n_{r}$ the refractive index of each component (skin or flesh). The uncertainty, $\Delta L_{i}$, linked to $L_{i}$ was evaluated to $1 \mathrm{~mm}$. The light input can be considered as a pulsed source of short duration $n_{r} \Delta L_{i} / c=4.6 \mathrm{ps}$. Therefore, each generated output time-dependent signal can be assimilated to the impulse response of the simulated model. The source-detector distance on the periphery of a sphere of radius $r_{s}$ may be defined by the curvilinear distance $r_{s} \theta$ or by the projected radial distance $\rho=r_{s} \sin \theta$. Each defined source-detector distance can be easily computed for a given source-detector angle $\theta$. Unless otherwise specified, the source detector distance used in the simulations (Section 3) corresponds to the projected radial distance $\rho=r_{s} \sin \theta$.

The Monte Carlo model was developed on the base of the free software SCILAB, and implemented on a Personal Computer (Processor Intel Core i5, $2.60 \mathrm{GHz}$ ). Globally, the simulations were run with $\sim 10^{5}$ to $\sim 4 \times 10^{6}$ photons, thus depending on the absorption coefficient of the considered medium. 
Table 1

Optical parameters of the apple flesh reported by authors who worked with steady-state hyper spectral method (SSM) or with time-resolved method (TRS).

\begin{tabular}{|c|c|c|c|c|}
\hline Apple varieties & $\mu_{s}^{\prime(f)}\left(\mathrm{mm}^{-1}\right)$ & $\mu_{a}^{(\mathrm{f})}\left(\mathrm{mm}^{-1}\right)$ & Wavelength range $(\mathrm{nm})$ & References \\
\hline Golden Delicious & $\begin{array}{l}\sim 1.2^{\min }-1.5^{\max } \\
\sim 2.1\end{array}$ & $\begin{array}{l}0.003-0.01,0.01^{*} \\
0.0025-0.0075\end{array}$ & $\begin{array}{l}600-700,675^{*} \\
700-900\end{array}$ & $\begin{array}{l}\text { Cubeddu et al. (2001a) (TRS) } \\
\text { Cubeddu et al. (2001b) (TRS) }\end{array}$ \\
\hline Golden and Red Delicious Bruised & $\begin{array}{l}1.1^{\min }-1.9^{\max } \\
\sim 0.45-1 \\
\sim 0.5 \text { to } \sim 0.8\end{array}$ & $\begin{array}{l}\sim 0.001-0.03,0.03^{*} \\
\sim 0.01 \text { to } \sim 0.04 \\
\sim 0.001-0.01\end{array}$ & $\begin{array}{l}500-950,675^{*} \\
750-900 \\
700-900\end{array}$ & $\begin{array}{l}\text { Lu et al. (2006) (SSM) } \\
\text { Qin et al. (2009) (SSM) } \\
\text { Lu et al. (2010) (SSM) }\end{array}$ \\
\hline Braeburn & $\begin{array}{l}\sim 1.2 \\
1.6-2 \\
\sim 1 \text { to } \sim 1.3 \\
1.5-2\end{array}$ & $\begin{array}{l}\sim 0.04 \\
0.0037 \\
0.002-0.006 \\
0.02-0.01\end{array}$ & $\begin{array}{l}700-900 \\
790 \\
700-900 \\
700-900\end{array}$ & $\begin{array}{l}\text { Saeys et al. (2008) (SSM) } \\
\text { Vanoli et al. (2009) (TRS) } \\
\text { Vanoli et al. (2013) (TRS) } \\
\text { Trong et al. (2013) (SSM) }\end{array}$ \\
\hline Starking Delicious & $\begin{array}{l}\sim 1.4-2.2 \\
\sim 2.2\end{array}$ & $\begin{array}{l}0.003-0.015,0.015^{*} \\
0.0025-0.0075\end{array}$ & $\begin{array}{l}600-700,675^{*} \\
700-900\end{array}$ & $\begin{array}{l}\text { Cubeddu et al. (2001a) (TRS) } \\
\text { Cubeddu et al. (2001b) (TRS) }\end{array}$ \\
\hline Granny Smith & $\begin{array}{l}0.8-1.6 \\
\sim 1.9 \\
\sim 1.3 \\
\sim 1.2\end{array}$ & $\begin{array}{l}0.002-0.025,0.025^{*} \\
0.0025-0.0075 \\
\sim 0.04 \\
\sim 0.003\end{array}$ & $\begin{array}{l}600-700,675^{*} \\
700-900 \\
700-900 \\
750\end{array}$ & $\begin{array}{l}\text { Cubeddu et al. (2001a) (TRS) } \\
\text { Cubeddu et al. (2001b) (TRS) } \\
\text { Saeys et al. (2008) (SSM) } \\
\text { Vanoli et al. (2009) (TRS) }\end{array}$ \\
\hline
\end{tabular}

$\left({ }^{*}\right)$ indicates the link between two values inside the Table.

\subsection{Optical properties of apples}

The optical parameters of the flesh and the skin (or peel) are listed in Tables 1 and 2, respectively, for different apple varieties. The data related to the flesh have been reported by authors who worked with hyperspectral steady-state method, (SSM), (Lu et al., 2006; Saeys et al., 2008; Qin et al., 2009; Lu et al., 2010; Nguyen DoTrong et al., 2013) or with Time-resolved reflectance spectroscopy, (TRS), (Cubeddu et al., 2001a,b; Vanoli et al., 2009; Vanoli et al., 2013). As shown Table 1, the data well highlight the variation in optical properties observed between and within apples. They also suggest that the flesh optical properties are largely dependent on the maturity of the fruit, but are also sensitive to the measurement positions on the same apple and more simply to the technique which is used (SSM or TRS). The skin features listed Table 2 include histological data and optical properties. The thickness of the skin has been detailed by Homutová and Blažek (2006), Zamorskyi (2007), and Qin et al. (2009), whereas the thickness of the peel added to the cuticule has been measured by Verboven et al. (2013) and Konarska (2013). We note that the thickness of the apple skin ranges from $30 \mu \mathrm{m}$ to $\sim 180 \mu \mathrm{m}$, whereas the mean cuticule thickness amounted to $\sim 28 \mu \mathrm{m}$. Finally, according to Saeys et al. (2008), the absorption coefficient measured in two bulk layers: skin and flesh, was found to be much higher in skin tissue $\left(\sim 0.075 \mathrm{~mm}^{-1}\right)$ than in flesh tissue. Moreover, apple skin tissue was found to be approximate by three times (3-5 $\mathrm{mm}^{-1}$ ) more scattering than the flesh tissue. To our knowledge, other skin optical parameter sets have not been yet reported.

For both tissues, skin and flesh, the anisotropy factor $g$ was fixed at 0.8 . This value agrees with experimental data reported by Saeys et al. (2008) who showed that $g$ was about $0.6-0.7$ for some apple species, and also corresponds to the mean of measured values (0.7-0.9) for various biological tissues (Cheong et al., 1990). It is still interesting to note that varying $g$ in the range $0.7-0.9$ does not affect notably the Monte Carlo simulations (Pifferi et al., 1998; Alerstam, 2011).

\subsection{Tests and fitting procedures}

\subsubsection{Time-resolved mode}

The computational results related to the time-resolved mode were obtained in three steps.

(i) MC temporal profiles in homogeneous semi-infinite and spherical geometries were computed and compared with those derived from the solutions of the time-dependent diffusion equation for different source-detector distances.
(1) for the semi-infinite geometry we used the solution reported by Contini et al. (1997)

$F_{S e m}(\rho, t)=\frac{e^{-\mu_{a \frac{c}{n_{r}} t}}}{2\left(4 \pi D \frac{c}{n_{r}}\right)^{3 / 2}} t^{-5 / 2}\left[\frac{1}{\mu_{s}^{\prime}} e^{\frac{-r_{1}^{2}}{4 \frac{D_{n}}{n_{r}} t}}+\left(\frac{1}{\mu_{s}^{\prime}}+2 z_{b}\right) e^{\frac{-r_{2}^{2}}{4 \frac{D_{n}}{n_{r}} t}}\right]$

where $F_{\text {Sem }}(\rho, \mathrm{t})$ is the output flux from the medium, which is function of the time $t$ and of the source-detector radial distance $\rho, D=1 /\left[3\left(\mu_{a}+\mu_{s}^{\prime}\right)\right]$ is the diffusion coefficient, $z_{b}=2 A D$ is the distance between extrapolated and real boundaries, $c / n_{r}$ is the speed of light in the medium, and $A=\frac{1+R_{i}}{1-R_{i}}\left(R_{i}\right.$ represents the fraction of photons that is internally diffusively reflected, $R_{i}=0.493$ for $n_{r}=1.4$ see Haskell et al., 1994), $r_{1}^{2}=\left(\frac{1}{\mu_{s}^{\prime}}\right)^{2}+\rho^{2}$, and $r_{2}^{2}=\left(\frac{1}{\mu_{s}^{\prime}}+2 z_{b}\right)^{2}+\rho^{2}$.

(2) for a sphere of radius $r_{S}$ we used the solution given by Pogue and Patterson (1994)

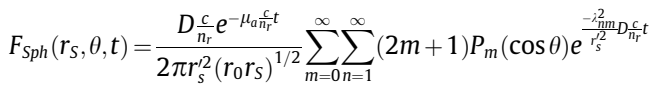

$$
\begin{aligned}
& \times \frac{J_{m+1 / 2}\left(\lambda_{m n} \frac{r_{0}}{r_{s}}\right)}{J_{m+3 / 2}\left(\lambda_{m n}\right)^{2}} \\
& \times\left[\left\{\frac{\lambda_{m n}}{r_{S}^{\prime}}-\frac{(2 \mathrm{~m}+3) m r_{S}^{\prime}}{\lambda_{m n} r_{S}^{2}}\right\} J_{m+3 / 2}\left(\lambda_{m n} \frac{r_{S}}{r_{S}^{\prime}}\right)+\frac{m}{r_{S}} J_{m+5 / 2}\left(\lambda_{m n} \frac{r_{S}}{r_{S}^{\prime}}\right)\right]
\end{aligned}
$$

where $r_{0}=r_{S}-\frac{1}{\mu_{s}^{\prime}}$, and $r_{S}^{\prime}=r_{S}+2 \mathrm{AD}$ is the extrapolated radius. Here $P_{m}(x)$ are the Legendre polynomials of order $m$, $\theta$ the source-detector angle, and $\lambda_{m n}$ are the positive roots of the Bessel functions of the first kind $J_{m+1 / 2}(\mathrm{x})$.

(ii) MC simulations were run for a simplified spherical geometry with different radii, where the skin and flesh were assigned to the same optical properties. This tissue arrangement offers some guidelines to examine the applicability of the simple solution of the semi-infinite model (Eq. (1)) in fitting procedures to retrieve the optical properties of the flesh, in case of a spherical geometry.

To retrieve the optical properties of the flesh we used two different fitting procedures. The first is based on the determination of $\mu_{s}^{\prime}$ from the ratio of two intensity measurements $F\left(\rho_{1}, t\right)$ and $F\left(\rho_{2}, t\right)$ acquired at two source-detector radial distances $\rho_{1}$ and $\rho_{2}\left(\rho_{2}>\rho_{1}\right)$ during the same time delay $t$. According to (Martelli et al., 2000), this is accomplished by the use of the relationship 
Thickness and optical properties of the apple skin reported by several authors.

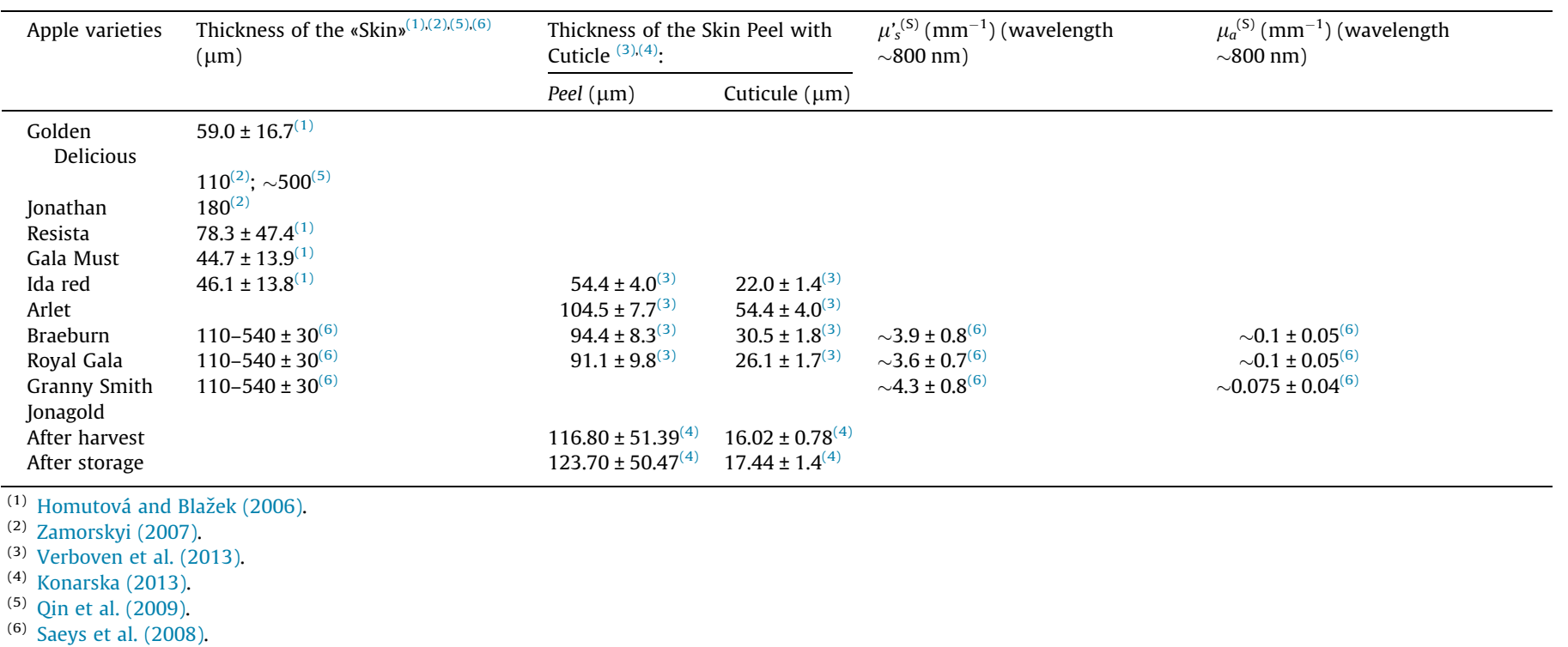

$\mu_{s}^{\prime} \approx \frac{4 c t\left(\rho_{2}^{2}-\rho_{1}^{2}\right)}{3 n_{r} \ln \left[F\left(\rho_{1}, t\right) / F\left(\rho_{2,} t\right)\right]}$

if $\mu_{a} \ll \mu_{s}^{\prime}$. The derived $\mu_{s}^{\prime}$ values are then used as prior information to initiate the Levenberg-Marquardt algorithm and consequently to retrieve the absorption coefficient $\mu_{a}$. The second fitting procedure uses the Levenberg-Marquardt algorithm to arrive iteratively at the best estimates of $\mu_{a}$ and $\mu_{s}^{\prime}$ from the temporal MC simulations, $F(\rho, t)$.

(iii) MC simulations were performed for the two-layer spherical geometry, in order to quantify the errors in the retrieved optical properties of the flesh, as functions of the sphere radii, skin thickness, skin optical properties and sourcedetector distances.

\subsubsection{Spatially-resolved mode}

As a second interest we have also focused on the retrieval of internal optical properties from the spatially resolved light intensity caused by the curved apple surface. For this, the apple was still considered as a simple homogeneous sphere or as a two-layer spherical model including the skin. The data analysis procedure refers to the steady-state diffusion theory model $R(\rho)$ previously described by Farrel et al. (1992),

$R(\rho)=\frac{a^{\prime}}{4 \pi}\left[\frac{1}{\mu_{t}^{\prime}}\left(\mu_{e f f}+\frac{1}{r_{1}}\right) \frac{e^{-\mu_{e f f} r_{1}}}{r_{1}^{2}}+\left(\frac{1}{\mu_{t}^{\prime}}+2 z_{b}\right)\left(\mu_{e f f}+\frac{1}{r_{2}}\right) \frac{e^{-\mu_{e f f} r_{2}}}{r_{2}^{2}}\right]$

where $r_{1}=\left[\left(\frac{1}{\mu_{t}^{\prime}}\right)^{2}+\rho^{2}\right]^{1 / 2}, r_{2}=\left[\left(\frac{1}{\mu_{t}^{\prime}}+2 z_{b}\right)^{2}+\rho^{2}\right]^{1 / 2}$, and $a^{\prime}=\mu_{s}^{\prime} / \mu_{t}^{\prime}$. This analytical equation predicts the spatially-resolved scattering profile at the surface of the semi-infinite medium, which is dependent upon source-detector radial distance $\rho$ and optical properties $\mu_{\text {eff }}$ and $\mu_{t}^{\prime}=\mu_{a}+\mu_{s}^{\prime}$.

To retrieve $\mu_{t}^{\prime}$ and $\mu_{\text {eff }}$ (and also $\mu_{a}=\frac{\mu_{e f f}^{2}}{3 \mu_{t}^{\prime}}$ and $\mu_{s}^{\prime}=\mu_{t}^{\prime}-\mu_{a}$ ), we again used two different fitting procedures based on Eq. (4). The first consists on the determination of $\mu_{\text {eff, by plotting } \ln \left(\rho^{2} R(\rho)\right)}$ against $\rho$, considering the slope of the curve for large $\rho$ (Farrel et al., 1992). The data obtained are then used as prior information to initiate a Levenberg-Marquardt algorithm to extract $\mu_{t}^{\prime}$. The second procedure allows to retrieve $\mu_{\text {eff }}$ and $\mu_{t}^{\prime}$ by fitting the normalized reflectance data with a Levenberg-Marquardt algorithm.

All above mentioned fitting procedures (Sections 2.3.1 and 2.3.2) were conducted with the Least squares routine solver of SCILAB (Isqrsolve function).

Table 3 lists the whole fitting procedures related to the time-resolved mode (Eqs. (1) and (3)) and spatially-resolved mode (Eq. (4)), which have been performed on spherical apple geometries with and without skin layer.

Note that Eqs. (1), (3) and (4) have been established under the assumption of the diffusion theory. This involves to locate the source at depth $1 / \mu_{s}^{\prime}$, below the physical tissue surface and set the zero-fluence boundary at a certain height above the surface $z_{b}=2 A D$ (Extrapolated boundary conditions EBC).

It should be emphasized that the validity of diffusion theory is limited to cases of tissues where the light has been highly scattered. This effect depends on the average distance between scattering centres $1 / \mu_{s}^{\prime}$, on the optical albedo $a^{\prime}=\mu_{s}^{\prime} /\left(\mu_{s}^{\prime}+\mu_{a}\right)$, and on the source-detector geometries.

Then, the fundamental requirement for diffusion theory is that $\mu_{a} \ll \mu_{s}^{\prime}$. This condition is well fulfilled in the database shown Table 1. It is also preferable to consider light far away from the source, or to disregard a large part of the leading edge of the recorded temporal data (Pifferi et al., 1998). This latter point will be discussed below.

\section{Results and discussion}

\subsection{Validation of Monte Carlo simulations}

Fig. 2(a) and (b) compare time-resolved reflectance solutions, $F_{\text {Sem }}(\rho, t)$ and $F_{\mathrm{Sph}}\left(r_{S}, \theta, t\right)$ respectively, with Monte Carlo simulations performed from a sphere radius $r_{S}=1000 \mathrm{~mm}$ (assimilated to a semi-infinite medium) and a sphere of radius $30 \mathrm{~mm}$. The curves were generated for three source-detector radial distances $\rho=7 \mathrm{~mm}, 11 \mathrm{~mm}$, and $15 \mathrm{~mm}$ (Fig. 2(a)), and for three curvilinear source-detector distances $S-D=7 \mathrm{~mm}, 11.25 \mathrm{~mm}$, and $15.7 \mathrm{~mm}$ (Fig. 2(b)), with the following optical parameters $\mu_{a}=0.01 \mathrm{~mm}^{-1}$, $\mu_{s}^{\prime}=1 \mathrm{~mm}^{-1}$ and $n_{r}=1.4$. Monte Carlo temporal data were successfully fitted by the relationship (Kienle and Patterson, 1996) $\ln (F(t))=\mathrm{A}_{0}+A_{1} \ln t+A_{2}(\ln t)^{2}+\cdots+A_{n}(\ln t)^{n}$, which has the main 
Table 3

General view of fitting procedures related to the time-resolved mode (Eqs. (1) and (3)) and spatially-resolved mode (Eq. (4)), which have been performed on spherical apple geometries with and without skin layer.

\begin{tabular}{|c|c|c|c|c|c|c|}
\hline \multirow[t]{2}{*}{$\mu_{s}^{\prime(\mathrm{f})}\left(\mathrm{mm}^{-1}\right)$} & \multirow[t]{2}{*}{$\mu_{a}^{(\mathrm{f})}\left(\mathrm{mm}^{-1}\right)$} & \multirow[t]{2}{*}{ Sphere radius $r_{s}(\mathrm{~cm})$} & \multirow[t]{2}{*}{ Skin ${ }^{*}$ thickness $(\mu \mathrm{m})$} & \multicolumn{2}{|c|}{ TRS fittings } & \multirow[t]{2}{*}{ SSM fittings Eq. (4) } \\
\hline & & & & Eq. (1) & Eq. (3) & \\
\hline \multirow[t]{2}{*}{1} & 0.01 & $100 ; 50 ; 30 ; 20 ; 10 ; 6 ; 5 ; 4 ; 3.5 ; 3 ; 2 ; 1.5 ; 1$ & $0 ; 55 ; 100 ; 140 ; 250 ; 500$ & Yes & Yes & Yes \\
\hline & 0.03 & $100 ; 50 ; 40 ; 30 ; 20 ; 15 ; 10 ; 7 ; 4$ & 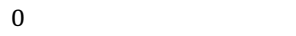 & No & Yes & No \\
\hline \multirow[t]{2}{*}{0.8} & 0.01 & $100 ; 30 ; 15 ; 10 ; 7 ; 4 ; 3 ; 2.5$ & 0 & No & Yes & No \\
\hline & 0.03 & $100 ; 50 ; 30 ; 20 ; 10 ; 8 ; 6 ; 4 ; \mathbf{3} ; 1.5$ & $0 ; 100$ & Yes & Yes & No \\
\hline \multirow[t]{2}{*}{0.6} & 0.01 & $100 ; 50 ; 30 ; 15 ; 10 ; 8 ; 7 ; 5 ; 4 ; 3$ & 0 & Yes & Yes & No \\
\hline & 0.03 & $100 ; 30 ; 20 ; 10 ; 7 ; 5 ; 4 ; 3 ; 2.5 ; 2$ & 0 & Yes & Yes & No \\
\hline 2 & 0.003 & $100 ; 30 ; 20 ; 10 ; 6 ; 4 ; \mathbf{3} ; 2.5 ; 1.5$ & $0 ; \mathbf{1 0 0}$ & Yes & Yes & No \\
\hline
\end{tabular}

Bold values indicate links between specific values used in computation. $\mu_{a}^{(S)}=0.05 \mathrm{~mm}^{-1}, \mu_{s}^{(S)}=4 \mathrm{~mm}^{-1}$.

(a)

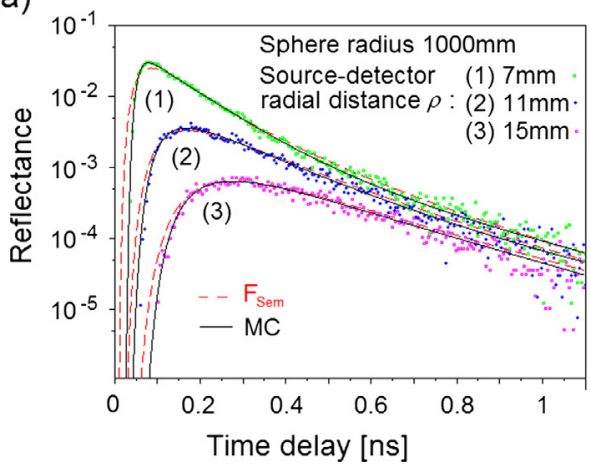

(b)

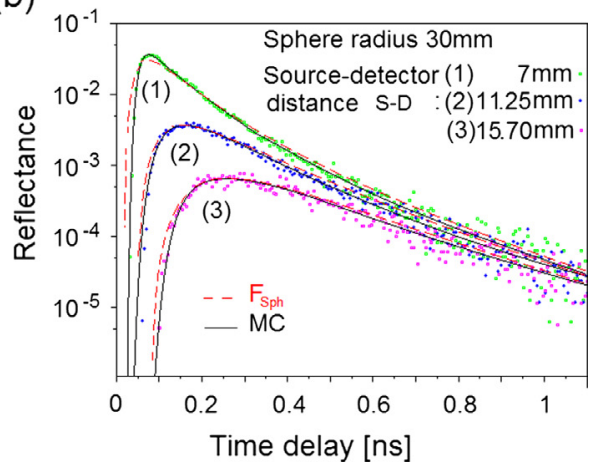

Fig. 2. (a) Comparison of time-resolved diffuse reflectance $F_{\text {Sem }}(\rho, t)$ with Monte Carlo simulations, calculated at three source-detector distances $\rho=7 \mathrm{~mm}$, $11 \mathrm{~mm}$, and $15 \mathrm{~mm}$ with $\mu_{a}^{(\mathrm{f})}=0.01 \mathrm{~mm}^{-1}, \mu_{s}^{(\mathrm{f})}=1 \mathrm{~mm}^{-1}$ and $n_{0}=1.4$. (b) Comparison of time-resolved diffuse reflectance $F_{\mathrm{Sph}}(\rho$, $t$ ) with Monte Carlo simulations, calculated for a sphere radius $r_{S}=30 \mathrm{~mm}$, at three curvilinear source-detector distances $S-D=7 \mathrm{~mm}, 11.25 \mathrm{~mm}$, and $15.7 \mathrm{~mm}$ with $\mu_{a}^{(\mathrm{f})}=0.01 \mathrm{~mm}^{-1}, \mu_{s}^{\prime(\mathrm{f})}=1 \mathrm{~mm}^{-1}$ and $n_{r}=1.4$.

advantage to smooth noisier data at large time values. As expected (Alerstam, 2011), we note in each figure that the deviation from the Monte Carlo simulations occurs at early times. This shows that early-arriving photons are likely not to be described by a model based on the diffusion approximation (with EBC). For longer times, it can be observed that there is better agreement between Monte Carlo simulations and diffusion results. Nonetheless, for the case of the spherical sample (Fig. 2(b)) good convergence of temporal profiles at longer photon flight times was obtained by increasing the number of Bessel functions up to 160 .

These findings show that in the fitting procedures based on diffusion models it is necessary to chose a fitting range that discards the early photons of Monte Carlo temporal data.

\subsection{Apple considered as a homogeneous spherical medium}

\subsubsection{Effect of the sphere radius}

In this sub-section we considered the apple as a simplified spherical geometry comprising the flesh with several radii $\left(10 \mathrm{~mm}<r_{S}<1000 \mathrm{~mm}\right)$. The temporal MC simulations were performed using the following optical parameter sets $\mu_{s}^{(\mathrm{f})}=0.6$; 0.8; 1 and $2 \mathrm{~mm}^{-1}, \mu_{a}^{(\mathrm{f})}=0.03 ; 0.01 ; 0.003 \mathrm{~mm}^{-1}$, whose combination covers a large range of optical properties of different apple varieties (see Table. 1). To compute $\mu_{s}^{\prime}$ from Eq. (3) (first fitting procedure) time delay values were chosen such as these are positioned farther on the peak intensity of both time-dependent signals $F\left(\rho_{1}, t\right)$ and $F\left(\rho_{2}, t\right)$, preferentially in the falling edge of each signal. Then, the method has the advantage to explore the regions where multiple scattering occurs, even if the source-detector distances are not too large. Moreover, ten time delay values were considered to obtain a set of $\mu_{s}^{\prime}$, but only the averaged value of $\mu_{s}^{\prime}$ was retained in the fitting procedure. The results computed for $\rho_{1}=3 \mathrm{~mm}$ and $\rho_{2}=7 \mathrm{~mm}$ are shown in Fig. 3(a), whereas the corresponding retrieved $\mu_{a}$ values are displayed Fig. 4. To test the dependence of the $\mu_{s}^{\prime}$ value on the source-detector distances $\rho_{1}$ and $\rho_{2}$, other simulations were performed using $\rho_{1}=5 \mathrm{~mm}-$ $\rho_{2}=7 \mathrm{~mm}$ and $\rho_{1}=7 \mathrm{~mm}-\rho_{2}=11 \mathrm{~mm}$. No systematic improvement on the averaged $\mu_{s}^{\prime}$ value presented Fig. 3(a) was found, as long as the time delay values are positioned on the falling part of both $F\left(\rho_{1}, t\right)$ and $F\left(\rho_{2}, t\right)$ curves. The second fitting procedure uses the Levenberg-Marquardt algorithm to arrive iteratively at the best estimates of $\mu_{a}$ and $\mu_{s}^{\prime}$ from the temporal MC simulations, $F(\rho, t)$. The time range for the nonlinear regression was chosen as follows: - the start time was defined as the time to reach $70 \%$ of $F_{\max }(\rho, t)$; - the end time was the time at which $F(\rho, t)$ has decreased up to $F_{\max }(\rho, t) / 100$, where $F_{\max }$ is the maximum value of the reflectance curve. An initial guess of $\mu_{s}^{\prime}=4 \mathrm{~mm}^{-1}$ and $\mu_{a}=0.005 \mathrm{~mm}^{-1}$ was appropriate for reaching the best fit of $\mu_{s}^{\prime}$ and $\mu_{a}$ in most cases. The corresponding results obtained for $\rho=7 \mathrm{~mm}$ are shown in Figs. 3(b) and 5. Note that in Figs. 3-5, the gray bar delineates the radii of 30 and $40 \mathrm{~mm}$ which are often encountered with apple varieties.

As shown in Fig. 3(a) and (b), the errors involved on the retrieval of $\mu_{s}^{\prime}$ decrease as the sphere (apple) size is increased. Considering the smaller spherical geometry investigated $\left(r_{S}=10 \mathrm{~mm}\right)$, the departures from the true $\mu_{s}^{\prime}$ values are typically about: $25 \%$ (Fig. 3(a)) and $8 \%$ (Fig. 3(b)) for $0.6 \mathrm{~mm}^{-1}, 19 \%$ (Fig. 3(a)) and $12 \%$ (Fig. 3(b)) for $0.8 \mathrm{~mm}^{-1}, 14 \%$ for $1 \mathrm{~mm}^{-1}$, and $12 \%$ for $2 \mathrm{~mm}^{-1}$. Above $r_{S}=100 \mathrm{~mm}$, and up to $1000 \mathrm{~mm}$, errors are less than $2 \%$, and the retrieved $\mu_{s}^{\prime}$ values are not very influenced by the sphere radius. Globally, a similar trend is shown Fig. 3(a) and (b), especially when $\mu_{s}^{\prime}>0.6 \mathrm{~mm}^{-1}$, whatever the fitting procedure may be. However, the dispersions of the retrieved $\mu_{s}^{\prime}$ values are mainly caused by the uncertainties of the MC 

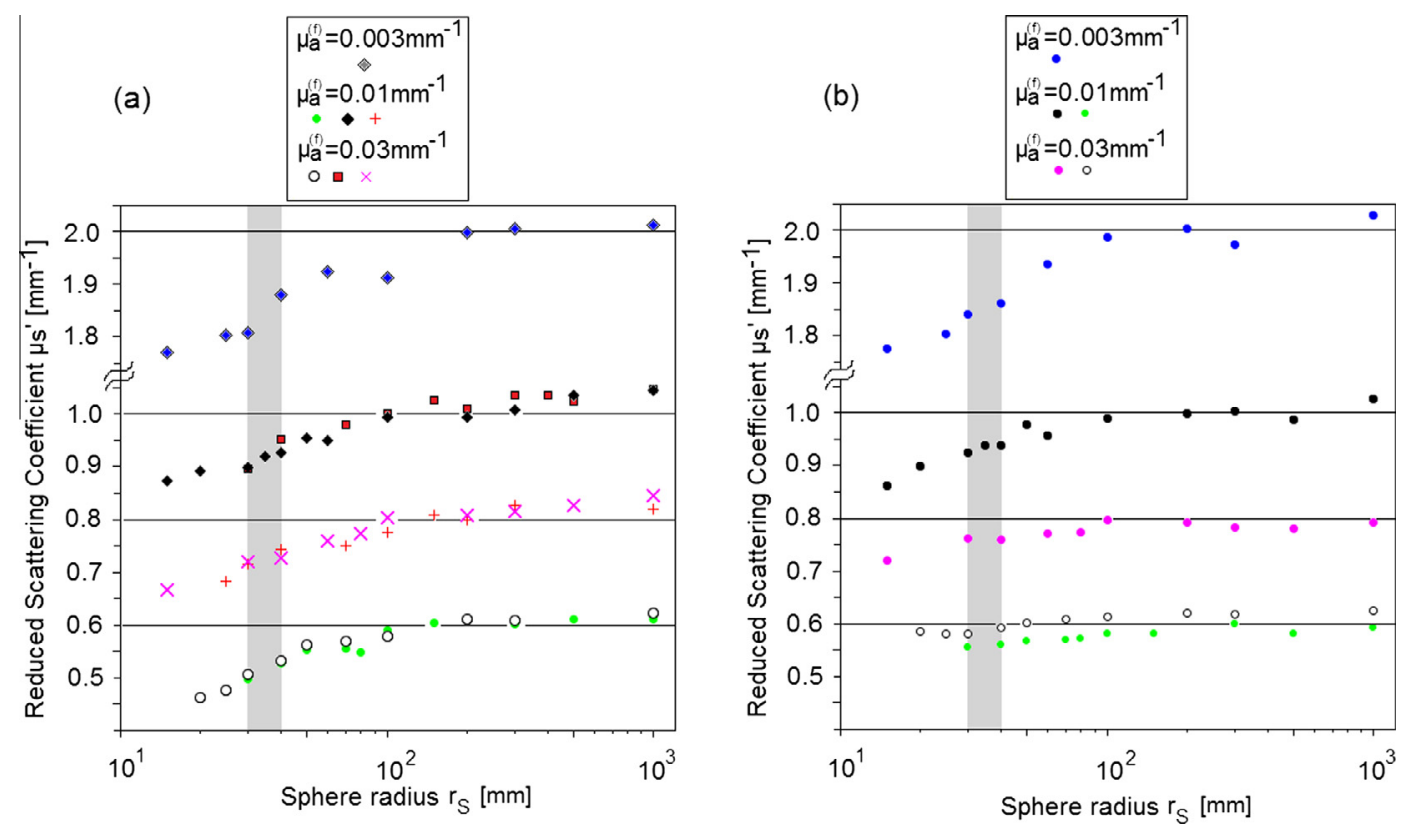

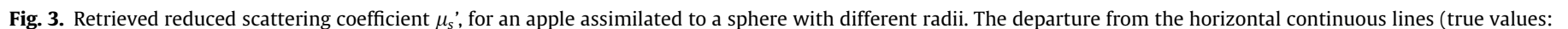

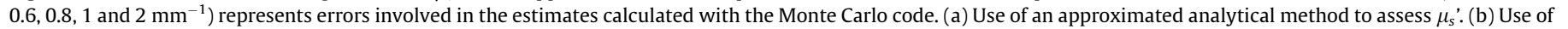
a Levenberg-Marquardt fitting algorithm.

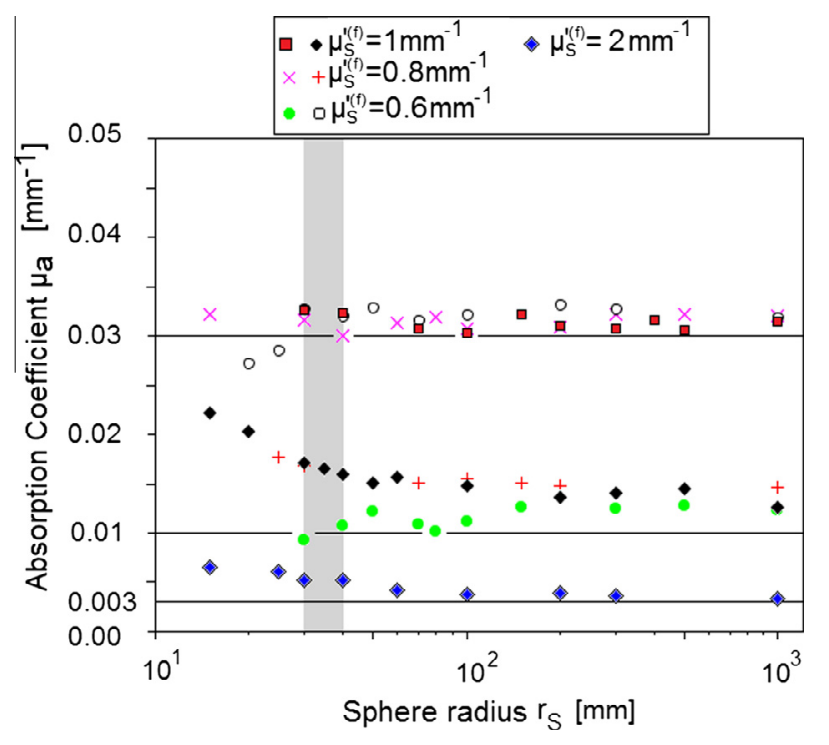

Fig. 4. Retrieved absorption coefficient $\mu_{a}$, for a homogeneous sphere with different radii using prior $\mu_{s}^{\prime}$ information to initiate a Levenberg-Marquardt algorithm. Monte Carlo data were generated with $\mu_{a}^{(\mathrm{f})}=0.003,0.01,0.03 \mathrm{~mm}^{-1}$ and $\mu_{s}^{(\mathrm{f})}=0.6$, $0.8,1$ and $2 \mathrm{~mm}^{-1}$. The departure from the horizontal continuous lines represents error in the estimates.

simulations. The corresponding retrieved $\mu_{a}$ values are shown in Figs. 4 and 5.

On the whole, the retrieved $\mu_{a}$ values using the first method (Fig. 4) appear less dependent on the sample radius than those obtained with the second procedure (Fig. 5). This can probably be explained by the fact that the first method is based on the assumption that $\mu_{a} \ll \mu_{s}^{\prime}$, which increases the errors in $\mu_{s}{ }^{\prime}$ and thus reduces by compensation the errors linked to the estimated $\mu_{a}$ values. In contrast, the retrieved $\mu_{a}$ values (Fig. 5) using the whole Levenberg-Marquardt algorithm (second fitting method) decrease progressively as a function of the sample radius. Interestingly, the trends, displayed Figs. 3(b) and 5, agree well with those reported

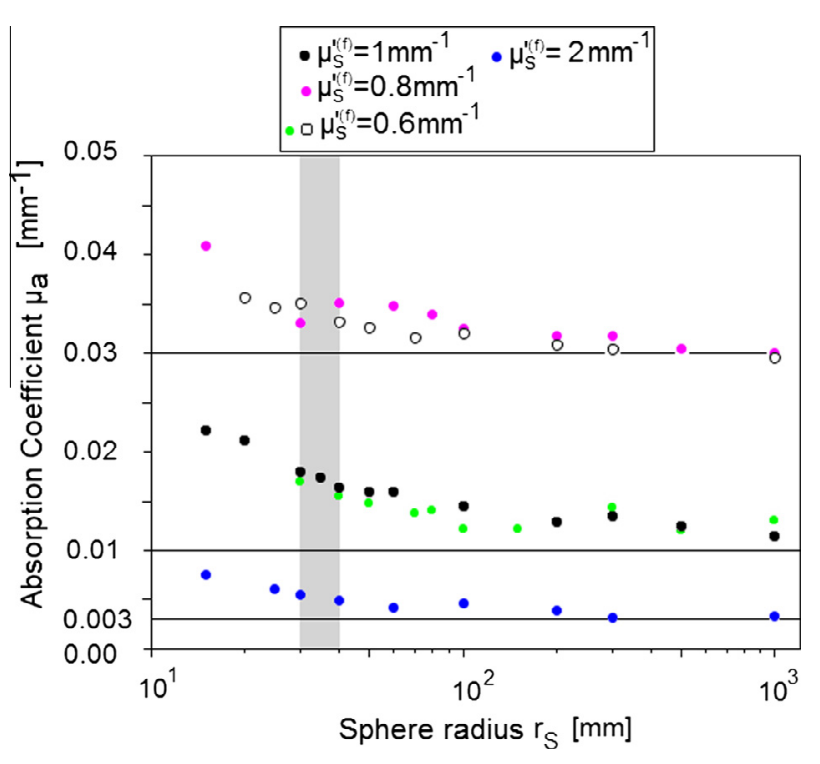

Fig. 5. Retrieved absorption coefficient $\mu_{a}$ for a homogeneous sphere with different radii using a fitting Levenberg-Marquardt procedure. The data were generated with $\mu_{a}^{(\mathrm{f})}=0.003,0.01,0.03 \mathrm{~mm}^{-1}$ and $\mu_{s}^{(\mathrm{f})}=0.6,0.8,1$ and $2 \mathrm{~mm}^{-1}$.

by Pogue and Patterson (1994) and Sassaroli et al. (2001) who studied the effects of the sample curvature, using frequency-resolved and time-resolved measurements, respectively.

\subsubsection{Effect of the source-detector distance}

The effect of varying the source-detector distances from $5 \mathrm{~mm}$ to $15 \mathrm{~mm}$ is shown in Fig. 6(a) and (b). All results were obtained using only the second fitting procedure. It is clear from Fig. 6(a), that the semi-infinite model (available for $r_{s}=1000 \mathrm{~mm}$ ) recovers the absorption coefficient $\mu_{a}$ better than $10 \%$ for source-detector radial distances greater than $9-10 \mathrm{~mm}$, and the $\mu_{s}^{\prime}$ better than $5 \%$ for source-detector distances in the range $7-15 \mathrm{~mm}$. 
(a)

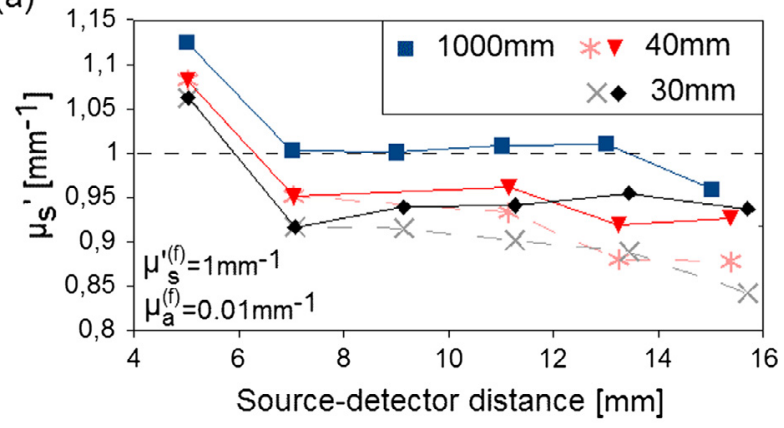

(b)

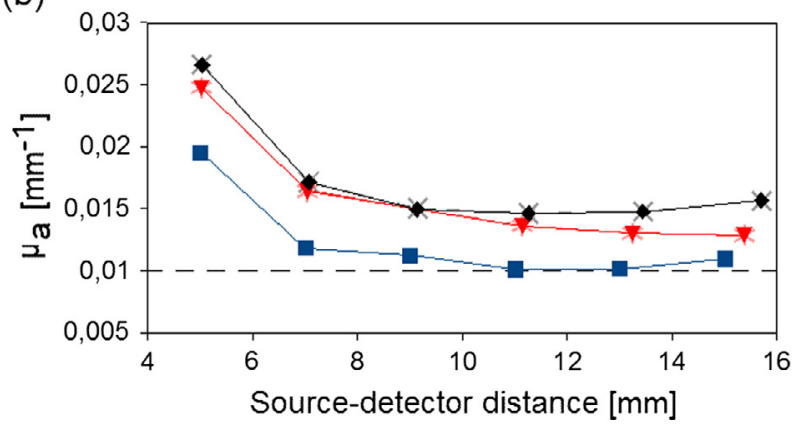

Fig. 6. Retrieved optical properties found at several source-detector distances by fitting (Levenberg-Marquardt) Monte Carlo data generated for a sphere with $r_{S}=30$, 40 and $1000 \mathrm{~mm} ; \mu_{a}^{(\mathrm{f})}=0.01 \mathrm{~mm}^{-1}, \mu_{s}^{(\mathrm{f})}=1 \mathrm{~mm}^{-1}$. In each figure, the departure from the horizontal dashed line represents errors in the estimates. Star and cross symbols indicate the results obtained using the curvilinear distances $r_{s} \cdot \theta$, instead of $\rho=r_{s} \cdot \sin \theta$ in the retrieval procedures. (a) absorption coefficient $\mu_{a}$, (b) reduced scattering coefficient $\mu_{s}^{\prime}$.

However, the description of light propagation inside a spherical geometry based on a semi-infinite model appears inaccurate as the sphere radius is decreased $\left(r_{s}=40\right.$ and $\left.30 \mathrm{~mm}\right)$. Although a notable improvement is found in the retrieved optical properties when a specified source-detector radial distance of $15 \mathrm{~mm}$ is chosen (see Cubeddu et al., 2001; Toricelli et al., 2008), $\mu_{a}$ is overestimated by $\sim 50 \%$ and $\mu_{s}^{\prime}$ underestimated $\sim 8-10 \%$, for $r_{s}=30 \mathrm{~mm}$ or $40 \mathrm{~mm}$.

In Fig. 6(a) and (b), the detector position used in the fitting procedure was also computed along the curved surface of the spherical apple model $\left(r_{s}=30\right.$ and $\left.40 \mathrm{~mm}\right)$. As shown, no difference is found in the values of the retrieved $\mu_{a}$, but large errors in the reduced scattering coefficients are expected for curvilinear source-detector distances lesser than $7 \mathrm{~mm}$ and greater than $15.7 \mathrm{~mm}$. In this case, we found results similar to those of Sassaroli et al. (2001), who showed that an optimal range of the curvilinear source-detector distance is required to minimize the errors in $\mu_{s}^{\prime}$.

\subsubsection{Curved surface steady-state reflectance}

Spatial profiles were obtained by integrating the temporal data acquired over the full projected source-detector distances ranging from 1 to $29 \mathrm{~mm}$, by steps of $1 \mathrm{~mm}\left(r_{s}<30 \mathrm{~mm}\right)$ or $2 \mathrm{~mm}$ $\left(r_{s} \geqslant 30 \mathrm{~mm}\right)$. The use of the projected distance, i.e. the sourcedetector radial distance $\rho$, mimics the imaging arrangement previously described by Qin and Lu (2008). The transformed spatial profiles were then normalized with respect to the value defined at $3 \mathrm{~mm}$. The simulations were performed using $\mu_{s}^{\prime(\mathrm{f})}=1 \mathrm{~mm}^{-1}$ and $\mu_{a}^{(\mathrm{f})}=0.01 \mathrm{~mm}^{-1}$. An initial guess of $\mu_{t}^{\prime}=0.5 \mathrm{~mm}^{-1}$ and $\mu_{\text {eff }}=0.09 \mathrm{~mm}^{-1}$ was appropriate for reaching the best fit of $\mu_{t}^{\prime}$ and $\mu_{\text {eff }}$ in case of using the second fitting procedure. Figs. 7(a) and (b) and 8 display the fitting results obtained for both

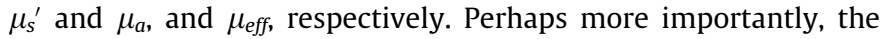
previous trends (see Section 3.2.1) in $\mu_{a}$ and $\mu_{s}^{\prime}$ are shown Fig. 7(a) and (b). However, the $\mu_{a}$ and $\mu_{s}^{\prime}$ values retrieved with the Levenberg-Marquardt algorithm (second procedure) are less dependent on the sphere radius than those assessed with the first fitting procedure (initiated Levenberg-Marquardt algorithm). This is mainly due to the inaccuracy of assessing $\mu_{\text {eff }}$ in case of low sphere radius (or large curvature), owing to boundary effects. The insert of the Fig. 8 shows that the determination of the slope $-\mu_{\text {eff }}$ remains more difficult as the radius of the sphere is decreased. This means that the individual estimates of $\mu_{\text {eff }}$ are more overestimated than those calculated from the retrieved $\mu_{a}$ and $\mu_{s}^{\prime}$ values through the relationship $\mu_{\text {eff }}=$ $\left[3 \mu_{a}\left(\mu_{a}+\mu_{s}^{\prime}\right)\right]^{1 / 2}$.

To avoid large distortion in the diffuse reflectance signals, the fitting range was next limited to $15 \mathrm{~mm}$, using a start distance of 1-3 mm with steps of $1 \mathrm{~mm}$. The corresponding retrieved values obtained with this second fitting procedure are shown in Fig. 7(a) and (b) for $\mu_{s}^{\prime}$ and $\mu_{a}$, respectively. It is clear that the errors related to both retrieved estimates are lesser $\left(2.5 \%-\mu_{s}^{\prime}\right.$, $20 \%-\mu_{a}$ ) than those obtained from the fitting limited to $29 \mathrm{~mm}$ $\left(5 \%-\mu_{s}^{\prime}, 35-50 \%-\mu_{a}\right)$, for a radius of $30 \mathrm{~mm}$. These findings are consistent with the results of Qin and Lu (2008), which showed the interest to fit the reflectance curves up to $10 \mathrm{~mm}$, in order to reduce the curvature effects of the apples.

\subsection{Apple considered as a two-layer spherical geometry}

In this sub-section, we investigate how the skin which surrounds the flesh of the apple affects the retrieved values of the internal optical properties. The Monte Carlo simulations, performed to answer to this debatable point, refer to tissue arrangements using steady-state and time-resolved spectroscopy methods. For this, we always used the second fitting procedure. As a general recipe, we start the fits using $\mu_{s}^{\prime}=4 \mathrm{~mm}^{-1}-\mu_{a}=0.005 \mathrm{~mm}^{-1}$ and $\mu_{t}^{\prime}=0.5 \mathrm{~mm}^{-1}-\mu_{\text {eff }}=0.09 \mathrm{~mm}^{-1}$ for the operations related to time-resolved mode and steady-state mode, respectively.

\subsubsection{Effects of the skin thickness}

Figs. 9(a) and 10 show the dependence of the normalized optical properties of the flesh as a function of the skin thickness. The retrieved absorption and reduced scattering coefficients are then divided by their initial values, using $\mu_{a 0}=0.0101 \mathrm{~mm}^{-1}$ $\mu_{s 0}^{\prime}=1.01 \mathrm{~mm}^{-1}$ (case of steady-state method), or $\mu_{a}^{(\mathrm{f})}=$ $0.01 \mathrm{~mm}^{-1}-\mu_{s}^{\prime(\mathrm{f})}=1 \mathrm{~mm}^{-1}$ (case of time-resolved method). The former optical parameter set corresponds to the values computed for $r_{s}=1000 \mathrm{~mm}$ in Fig. 7(a) and (b), whereas the second set refers to the true optical parameters of the flesh. The other optical parameters used in the simulations concern the apple skin for which $\mu_{a}^{(\mathrm{S})}=0.05 \mathrm{~mm}^{-1}$ and $\mu_{s}^{\prime}{ }^{(\mathrm{S})}=4 \mathrm{~mm}^{-1}$ (see Saeys et al., 2008). The skin thickness was varied from 0 to $140 \mu \mathrm{m}$, according to the data listed Table 2, and conveniently extended from $250 \mu \mathrm{m}$ to $500 \mu \mathrm{m}$ (see Qin and Lu, 2009; and Saeys et al., 2008).

The results obtained by simulating the steady-state reflectance curves (limited to $15 \mathrm{~mm}$ or $29 \mathrm{~mm}$ ) are shown in Fig. 9(a). Although the skin is a high diffusing layer medium, the ratio $\mu_{s}^{\prime} \mid \mu_{s o} o^{\prime}$ is practically insensitive to the skin thickness. Then, considering a realistic skin thickness of $100 \mu \mathrm{m}$ or even $180 \mu \mathrm{m}$, the maximum error in the retrieved $\mu_{s}^{\prime}$ values is on the order of $2.5 \%$ or $5 \%$, for an apple with a radius of $30 \mathrm{~mm}$. In the same conditions, the semi-infinite tissue arrangement $\left(r_{s}=1000 \mathrm{~mm}\right)$ exhibits an error on $\mu_{s}^{\prime}$ less than $2 \%$.

In contrast, the retrieved $\mu_{a}$ values depicted in Fig. 9(a) lead to an error of about $20 \%$ or $35 \%$ for an apple of radius $30 \mathrm{~mm}$ covered by a skin layer of thickness $100 \mu \mathrm{m}$ or $180 \mu \mathrm{m}$. As might be expected, the skin acts like a thin protective layer which can also 
(a)

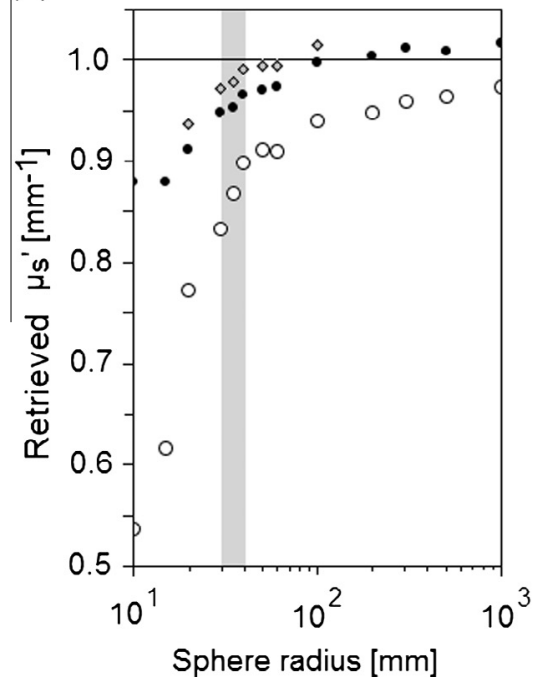

(b)

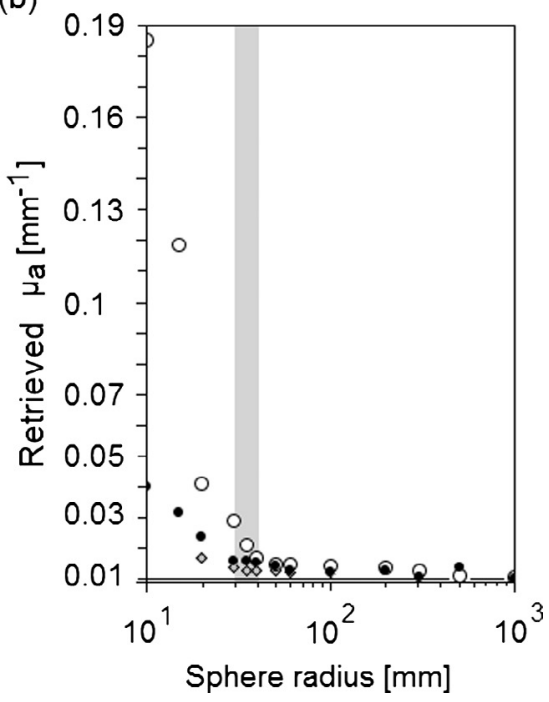

Fig. 7. Retrieved optical properties from Levenberg-Marquardt procedure (black and gray dots) or using prior $\mu_{\text {eff }}$ information to initiate a Levenberg-Marquardt algorithm (empty dots) to MC spatially-resolved reflectance data on sphere with several radii. The data were generated with $\mu_{a}^{(\mathrm{f})}=0.01 \mathrm{~mm}^{-1} ; \mu_{s}^{\prime(\mathrm{f})}=1 \mathrm{~mm} \mathrm{~m}^{-1}$, and acquired over the full source-detector range $\rho[1, \ldots, 29 \mathrm{~mm}]$ (round symbols), or $[1, \ldots, 15 \mathrm{~mm}]$ (diamond symbols) (a) reduced scattering coefficient $\mu_{\mathrm{s}}$ ' and (b) absorption coefficient $\mu_{a}$.

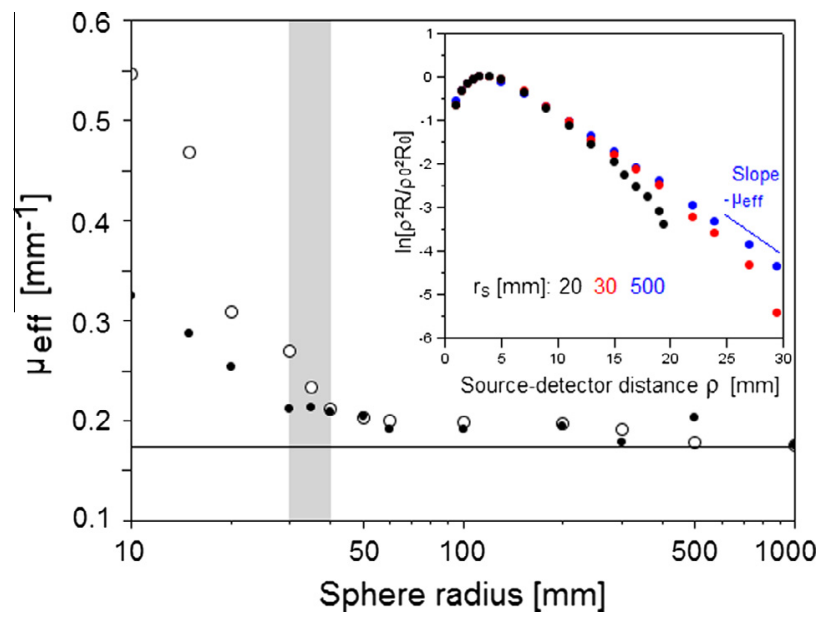

Fig. 8. Calculated effective absorption coefficient, $\mu_{e f f}$ from $\mu_{e f f}=\left[3 \mu_{a}\left(\mu_{a}+\mu_{s}{ }^{\prime}\right)\right]^{1 / 2}$, using retrieved $\mu_{a}$ and $\mu_{s}^{\prime}$ values corresponding to Fig. 7(a and b) (black dot) or by using the decreasing edge of $\log \left[\rho^{2} R(\rho)\right]$ (empty dot) versus $\rho$. The insert shows the plot of $\log \left[\rho^{2} R(\rho)\right]$ versus $\rho$ for a sphere having different radii: $r_{s}=10,30$ and $500 \mathrm{~mm}$. The curves were normalized by the value obtained at $\rho_{0}=3 \mathrm{~mm}$. The optical parameter $-\mu_{\text {eff }}$ corresponds to the slope of the curve for large $\rho$.

affect the amount of light which propagates in the flesh. This is illustrated in Fig. 9(b) and (c) where the isofluence lines are drawn in the equatorial plane of an apple $\left(r_{s}=30 \mathrm{~mm}\right)$ with and without skin (peeled apple). The thickness of the apple skin is successively fixed at $100 \mu \mathrm{m}$ (Fig. 9(b)) or at $55 \mu \mathrm{m}$ and $250 \mu \mathrm{m}$ (Fig. 9(c)). It is clear from the fluence patterns, that the thickness of the skin modulates the depth penetration of the light in the flesh. Moreover, it is also interesting to note that the simulations related to an apple of radius $30 \mathrm{~mm}$ with (Fig. 9(a)) and without skin (Fig. 7(a) and (b)) lead practically to the same errors in the $\mu_{s}^{\prime}(\sim 2.5-5 \%)$ and $\mu_{a}$ (20-35\%) estimates. This is consistent with the fact that the shape of the spatially resolved curve is minimally affected, except for points close to the source. However, the accuracy is largely dependent on the fitting range which was fixed for a generated reflectance curve. At this stage, a large amount of major pigments content (i.e., chlorophyll and anthocyanin) in apple skin cannot be ignored (Qin and Lu, 2008; Cubbedu et al., 2001). The presence of the apple skin is all the more evident because the sourcedetector distance fibre-optics is small (Nguyen DoTrong et al., 2014). Consequently, a greater absorption coefficient assigned to the skin layer in the Monte Carlo model could better demonstrate that steady-state measurements reflect the influence of both skin (surface) and flesh (sub-surface) tissues. This issue should be investigated in more detail in further studies.

The results obtained by simulating the time-resolved reflectance method are plotted in Fig. 10(a) and (b), for the spherical $\left(r_{s}=30 \mathrm{~mm}\right)$ and the semi-infinite $\left(r_{s}=1000 \mathrm{~mm}\right)$ geometries. Inspection of the data reveals similar trends to errors in $\mu_{a}$ and $\mu_{s}^{\prime}$ as those depicted in Fig. 9(a). However, the error in $\mu_{s}^{\prime}$ reaches about $10 \%$ for the above considered apple sample $\left(r_{s}=30 \mathrm{~mm}\right.$, skin thickness $=100 \mu \mathrm{m}$, source-detector distance $=7 \mathrm{~mm}$ ). Furthermore, the computations suggest that larger sourcedetector separations (relatively to $7 \mathrm{~mm}$ and $11 \mathrm{~mm}$ ) will provide better measurements of the flesh optical properties from an intact apple.

\subsubsection{Effects of the source detector spacing}

In Fig. 11(a) and (b), the effects upon the $\mu_{a}$ and $\mu_{s}^{\prime}$ estimates due to changing the source-detector spacing are examined for an apple of radius $30 \mathrm{~mm}$ and for a semi-infinite geometry $\left(r_{s}=1000\right.$ $\mathrm{mm}$ ). In both cases, the flesh tissue was assumed to be covered by a skin layer of thickness $100 \mu \mathrm{m}$, with $\mu_{a}^{(S)}=0.05 \mathrm{~mm}^{-1}$ and $\mu_{s}^{\prime}{ }^{(\mathrm{S})}=4 \mathrm{~mm}^{-1}$. The retrieved $\mu_{a}$ and $\mu_{s}^{\prime}$ values are still normalized by the true optical properties of the flesh, but three optical parameters sets were used to practically cover the variation range shown in Table 1: $\mu_{a}^{(\mathrm{f})}=0.01 \mathrm{~mm}^{-1}-\mu_{s}^{(\mathrm{f})}=1 \mathrm{~mm}^{-1}, \mu_{a}^{(\mathrm{f})}=0.03 \mathrm{~mm}^{-1}-$ $\mu_{s}^{\prime(\mathrm{f})}=0.8 \mathrm{~mm}^{-1}$, and $\mu_{a}^{(\mathrm{f})}=0.003 \mathrm{~mm}^{-1}-\mu_{s}^{\prime(\mathrm{f})}=2 \mathrm{~mm}^{-1}$. Two distinct trends are shown in Fig. 11(a) and (b). When the reduced scattering $\mu_{s}^{\prime(f)}$ (Fig. 11(a)) and absorption $\mu_{a}^{(\mathrm{f})}$ (Fig. 11(b)) coefficients of the flesh are lesser than the corresponding values of the skin, the estimates decrease rapidly as a function of the sourcedetector separation (from 5 to $7 \mathrm{~mm}$ ), and then tend to get more stabilized (from $7 \mathrm{~mm}$ to $15 \mathrm{~mm}$ ). In contrast, when the optical properties of the flesh are close to those of the skin $\left(\mu_{a}^{(\mathrm{f})}=0.03-\right.$ $\mathrm{mm}^{-1}$ or $\mu_{s}^{\prime(\mathrm{f})}=2 \mathrm{~mm}^{-1}$ ) the estimates slightly decrease as a function of the source-detector distance (from 5 to $15 \mathrm{~mm}$ ). In both cases, we note that the accuracy of the measurements is better with a source-detector distance of $15 \mathrm{~mm}$.

Fig. 12(a) and (b) depict the spatial sensitivity profiles for an apple model $\left(r_{s}=30 \mathrm{~mm}\right)$, at source-detector distances of $7 \mathrm{~mm}$ 
(b)
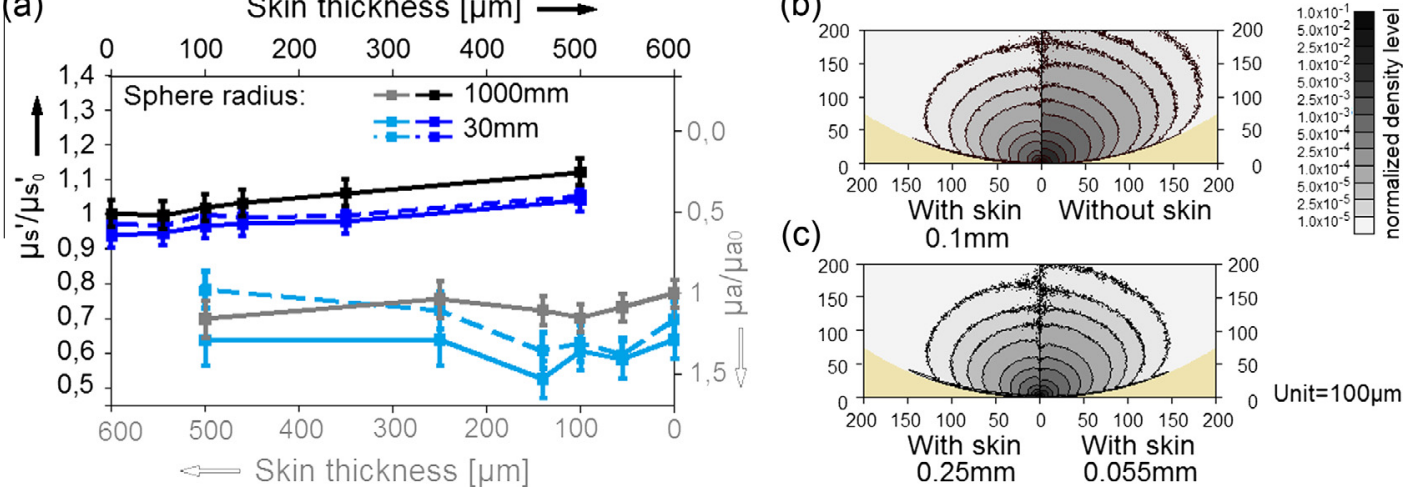

Fig. 9. (a) Effect of skin thickness on the assessment of the flesh optical properties using the steady-state reflectance method. Fitting range limited to 29 mm, grey and cyan symbols correspond to the normalized absorption coefficient, whereas black and blue ones correspond to the normalized reduced scattering coefficient. Fitting range limited to $15 \mathrm{~mm}$, cyan dashed line (normalized absorption coefficient) and blue dashed line (normalized reduced scattering coefficient). (b) and (c) Light distribution inside a spherical apple of radius $30 \mathrm{~mm}$, with and without skin: (b) skin thickness $100 \mu \mathrm{m}$, (c) large $(250 \mu \mathrm{m})$ or small (55 $\mu \mathrm{m})$ skin thickness. The maps were generated with $\mu_{a}^{(\mathrm{S})}=0.05 \mathrm{~mm}^{-1}, \mu_{s}^{\prime(\mathrm{S})}=4 \mathrm{~mm}^{-1}, \mu_{a}^{(\mathrm{f})}=0.01 \mathrm{~mm}^{-1}, \mu_{s}^{\prime(\mathrm{f})}=1 \mathrm{~mm}^{-1}$. (For interpretation of the references to colour in this figure legend, the reader is referred to the web version of this article.)

\begin{tabular}{|c|c|c|c|c|c|}
\hline$\longrightarrow$ & $\begin{array}{l}\text { Sphere } \\
\text { radius } \\
1000 \mathrm{~mm}\end{array}$ & $\begin{array}{l}\text { Source-detector } \\
\text { radial distance } \\
11 \mathrm{~mm}\end{array}$ & $-\bullet$ & $\begin{array}{l}\text { Sphere } \\
\text { radius } \\
1000 \mathrm{~mm}\end{array}$ & $\begin{array}{l}\text { Source-detector } \\
\text { radial distance } \\
7 \mathrm{~mm}\end{array}$ \\
\hline$\rightarrow-$ & $30 \mathrm{~mm}$ & $11 \mathrm{~mm}$ & - - & $30 \mathrm{~mm}$ & \\
\hline
\end{tabular}

(a)

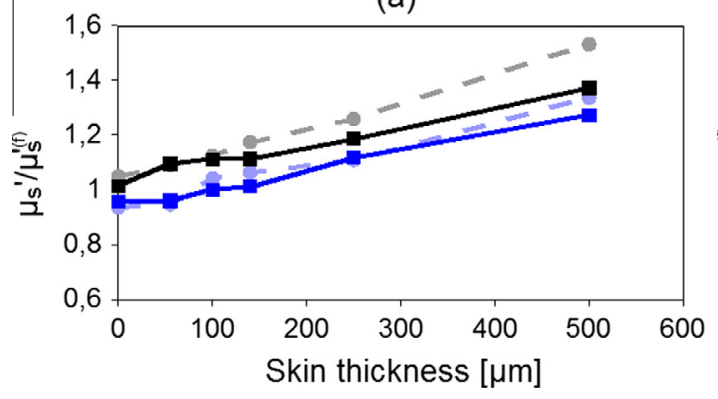

(b)

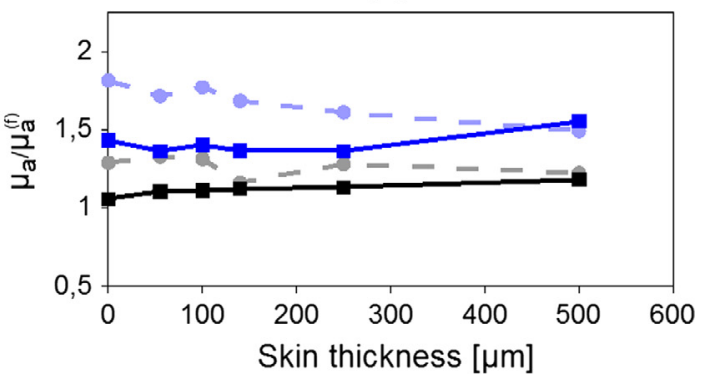

Fig. 10. Effect of skin thickness on the assessment of the flesh optical properties using time-resolved spectroscopy method. The data were computed for two spheres with radius $1000 \mathrm{~mm}$ and $30 \mathrm{~mm}$, respectively, and two source-detector radial distances $11 \mathrm{~mm}$ and $7 \mathrm{~mm}$. The flesh optical property set is $\mu_{a}^{(\mathrm{f})}=0.01 \mathrm{~mm}^{-1}$ and $\mu_{s}^{\prime(\mathrm{f})}=1 \mathrm{~mm}^{-1}$. (a) Relative absorption coefficient and (b) relative reduced scattering coefficient.

(a)

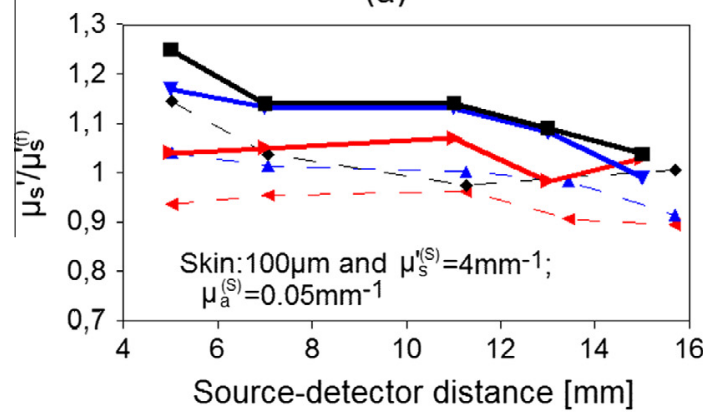

(b)

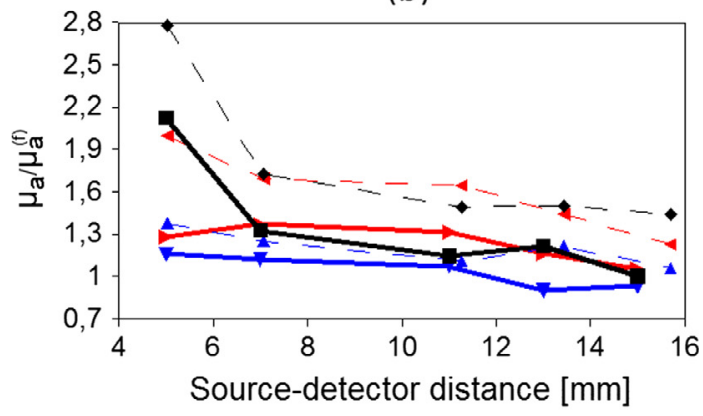

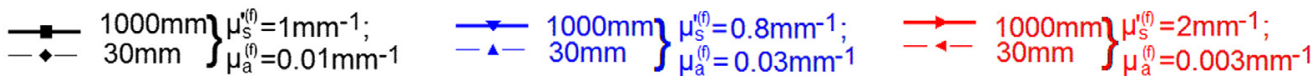

Fig. 11. Effect of source-detector distance on the assessment of the flesh optical properties using time-resolved spectroscopy method. The data were computed for two spheres with radius $1000 \mathrm{~mm}$ and $30 \mathrm{~mm}$, using skin thickness: $100 \mu \mathrm{m}, \mu_{a}^{(\mathrm{S})}=0.05 \mathrm{~mm}^{-1}, \mu_{s}^{(\mathrm{S})}=4 \mathrm{~mm}^{-1}$, and three different flesh optical property sets: $1-\mu_{a}^{\left({ }^{(\mathrm{f})}\right.}=0.01$ $\mathrm{mm}^{-1}, \mu_{s}^{\prime(\mathrm{f})}=1 \mathrm{~mm}^{-1} ; 2-\mu_{a}^{(\mathrm{f})}=0.03 \mathrm{~mm}^{-1}, \mu_{s}^{\prime(\mathrm{f})}=0.8 \mathrm{~mm}^{-1} ; 3-\mu_{a}^{(\mathrm{f})}=0.003 \mathrm{~mm}^{-1}, \mu_{s}^{\prime(\mathrm{f})}=2 \mathrm{~mm}^{-1}$. (a) Relative absorption coefficient and (b) relative reduced scattering coefficient.

and $15 \mathrm{~mm}$. The contours were drawn for $1 \%$ and $2 \%$ fall from the maximum sensitivity point. These profiles are computed with $\mu_{a}^{(S)}=0.05 \mathrm{~mm}^{-1}, \mu_{s}^{\prime(S)}=4 \mathrm{~mm}^{-1}$, skin thickness $100 \mu \mathrm{m}$, $\mu_{a}^{(\mathrm{f})}=0.01 \mathrm{~mm}^{-1}$ and $\mu_{s}^{\prime(\mathrm{f})}=1 \mathrm{~mm}^{-1}$. We note that the spatial sensitivity profiles represent the well known banana-shaped pattern (Feng et al., 1995), and that the light penetrates deeper regions in the flesh if the source-detector spacing increases from $7 \mathrm{~mm}$ to $15 \mathrm{~mm}$. 
(a)

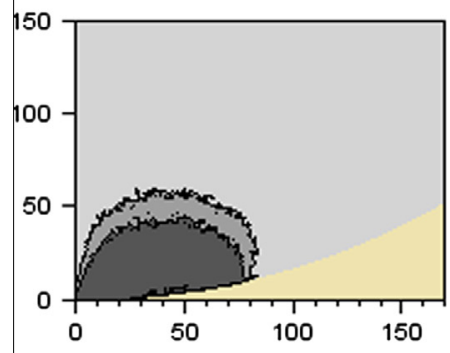

(b)

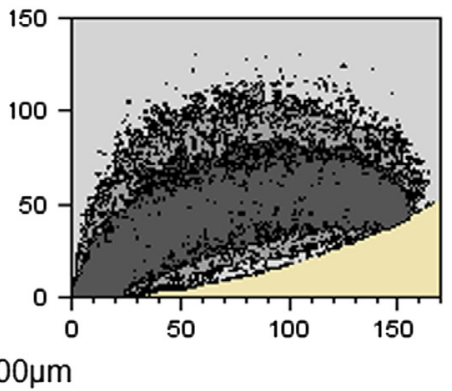

(d)

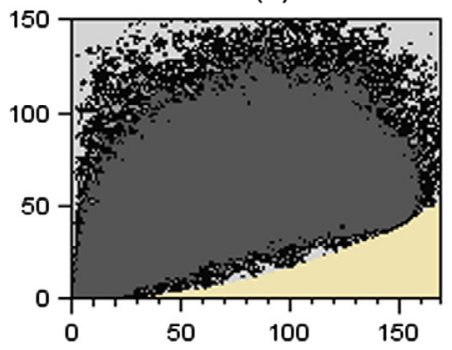

(f)

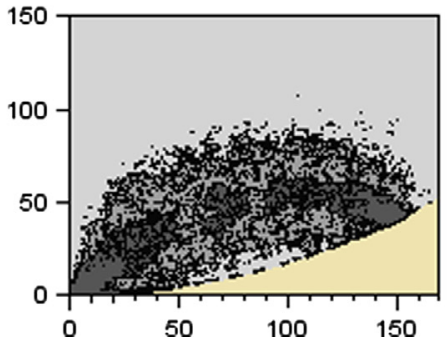

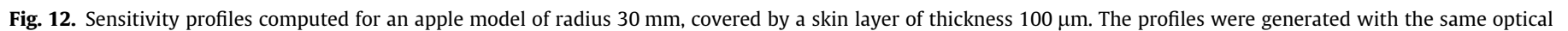
parameter sets as those used in Fig. 11. (a, c, e) Source-detector radial distance $\rho=7$ mm and (b, d, f) source-detector radial distance $\rho=15$ mm.

Table 4

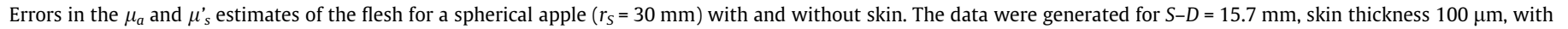
the same optical parameter sets of the flesh and skin as those used in Fig. 11.

\begin{tabular}{|c|c|c|c|c|c|c|}
\hline \multicolumn{3}{|c|}{$\begin{array}{l}\text { Optical parameters set of the flesh inside a sphere of radius } \\
r_{s}=30 \mathrm{~mm}:\end{array}$} & \multicolumn{2}{|c|}{$\begin{array}{l}\text { Optical parameters calculated at the } \\
\text { distance } S-D=15.7 \mathrm{~mm} \text { : }\end{array}$} & \multirow[t]{2}{*}{ Error in $\mu_{a}(\%)$} & \multirow[t]{2}{*}{ Error in $\mu_{s}^{\prime}(\%)$} \\
\hline$\mu_{a}^{(\mathrm{f})}\left(\mathrm{mm}^{-1}\right)$ & $\mu_{s}^{\prime(f)}\left(\mathrm{mm}^{-1}\right)$ & & $\mu_{a}\left(\mathrm{~mm}^{-1}\right)$ & $\mu_{\mathrm{s}}^{\prime}\left(\mathrm{mm}^{-1}\right)$ & & \\
\hline \multirow[t]{2}{*}{0.01} & 1 & With skin & $0.0145 \pm 0.0005$ & $1.01 \pm 0.01$ & 50 & 2 \\
\hline & & Without skin & 0.0155 & 0.94 & 55 & 6 \\
\hline \multirow[t]{2}{*}{0.003} & 2 & With skin & $0.0036 \pm 0.0001$ & $1.79 \pm 0.01$ & 23 & 10 \\
\hline & & Without skin & 0.0038 & 1.79 & 26 & 11 \\
\hline \multirow[t]{2}{*}{0.030} & 0.8 & With skin & $0.032 \pm 0.0005$ & $0.73 \pm 0.01$ & 8 & 8 \\
\hline & & Without skin & 0.0330 & 0.73 & 10 & 8 \\
\hline
\end{tabular}

When the flesh is considered as a highly scattering medium $\left(\mu_{s}^{\prime(\mathrm{f})}=2 \mathrm{~mm}^{-1}\right.$ and $\mu_{a}^{(\mathrm{f})}=0.003 \mathrm{~mm}^{-1}$ ), the influence of the skin on the light propagation seems reduced, whereas the spatial sensitivity profiles are less distinguishable from a banana's shape (Fig. 12(c) and (d)). Nevertheless, the penetration depth of the light inside the flesh increases from $10 \mathrm{~mm}$ to $15 \mathrm{~mm}$ as the sourcedetector distance increases from $7 \mathrm{~mm}$ to $15 \mathrm{~mm}$. Finally, when the flesh is absorbing $\left(\mu_{a}^{(\mathrm{f})}=0.03 \mathrm{~mm}^{-1}\right)$, with moderate scattering $\left(\mu_{s}^{\prime}(\mathrm{f})=0.8 \mathrm{~mm}^{-1}\right)$, the photon density remains high in the skin and the flesh for a source-detector equal to $7 \mathrm{~mm}$ (Fig. 12(e)). In spite of the absorption level, the light penetration in the flesh increases as the source-detector distance increases from $7 \mathrm{~mm}$ to $15 \mathrm{~mm}$ (Fig. 12(f)).
The accuracy of the time-resolved reflectance measurement for the assessment of $\mu_{a}$ and $\mu_{s}^{\prime}$ in a turbid medium (plane or curved) increases with the source-detector distance (Cubeddu et al., 1996, Sassaroli et al., 2001). Our results agree well with this observation, but they also show that measurements performed on apples with a source-detector of $15 \mathrm{~mm}$ is sensitive to the flesh optical properties (see Cubeddu et al., 2001; Torricelli et al., 2008). With this tissue-arrangement, the flesh is probed to a depth of about 10$15 \mathrm{~mm}$ (see Fig. 12(f), (b), and (d)), which seems reasonably in agreement with the rough value of $20 \mathrm{~mm}$ in the pulp, as reported by others (Cubeddu et al., 2001; Eccher Zerbini et al., 2002).

It is still interesting to compare the errors in $\mu_{a}$ and $\mu_{s}{ }^{\prime}$ estimates related to the flesh of an apple of radius $30 \mathrm{~mm}$ covered 
by a skin layer of thickness $100 \mu \mathrm{m}$ or without skin (peeled), when the source detector is fixed at $15 \mathrm{~mm}$. The results displayed in Figs. 11(a) and (b) and 6(a) and (b), and computed with $\mu_{a}^{(\mathrm{f})}=0.01 \mathrm{~mm}^{-1}-\mu_{s}^{(\mathrm{f})}=1 \mathrm{~mm}^{-1}$ show comparable errors in the estimates: $2 \%\left(\mu_{s}^{\prime}\right)-50 \%\left(\mu_{a}\right)$ for covered apple and $6 \%$ $\left(\mu_{s}^{\prime}\right)-55 \%\left(\mu_{a}\right)$ for a peeled apple. Other results (see Table 4 ), show that the errors in the estimates are systematically on the same order for intact and peeled apples.

As above discussed, the skin layer behaves like a thin absorbing-diffusing medium which contributes to attenuate the intensity of the re-emitted light at a given point of the surface. At the location of the detector ( $15 \mathrm{~mm}$ from the source), we verified that the shape of the computed re-emitted signals is minimally affected by the presence of the skin. Despite the fact that the skin layer is highly diffusing $\left(\mu_{s}^{\prime}(\mathrm{S})=4 \mathrm{~mm}^{-1}\right)$, these results strengthen the previous hypothesis formulated by Cubeddu et al. (2001), that the photons spend little time for travelling the skin, before being probed at the surface. In these circumstances, the absorption and scattering estimates of the flesh are very few influenced by the skin. Consequently, the errors linked to these estimates may be largely attributed to the finite curved boundary over which the measurement is accomplished. Improvements may be obtained, mainly in the $\mu_{a}$ estimates, if a mismatched curved geometry is used (see Eq. (2)) in the fitting procedure (Sassaroli et al., 2001). However, the price paid for this is essentially to increase the time needed for the convergence to the final values. On the whole, the time required for the fittings of the Monte Carlo data with the solution established for a semi-infinite geometry (Eq. (1)) does not exceed $1 \mathrm{~s}$.

\section{Conclusions}

The effects of the size and the skin of apples on the assessment of optical properties of the flesh have been investigated in this paper. To this end, apples have been modelled as a two layer spherical model with various radii, and comprising two distinct bulk layers such as skin and flesh. Monte Carlo simulations were then performed to mimic time-resolved reflectance measurements, or by integrating over time the data, steady-state reflectance measurements. In both cases, the absorption and reduced scattering coefficients were estimated from fitting the reflectance data with standard solutions of the diffusion approximation for a semi-infinite homogeneous medium.

When the apple is modelled as a simple homogeneous spherical structure (without skin), the finite curved boundary results in an overestimation of $\mu_{a}$ and underestimation of $\mu_{s}^{\prime}$ from their respective true values. The errors in the estimates tend to increase with decreasing the radius of the apple model. However, the errors in the $\mu_{a}$ estimates are always greater than those estimated for $\mu_{s}^{\prime}$. This may be due to the lost of photons at the curved boundary, an effect which is not accounted for in the semi-infinite diffusion equations used in the fitting procedures. For both optical spectroscopy modes (SSM and TRS), the source-detector(s) arrangement plays a major role on the error values linked to the estimated optical coefficients $\mu_{a}$ and $\mu_{s}^{\prime}$. Thus, a limited fitting range on the measured steady-state reflectance curve may reduce the light distortion due to apple curvature, whereas a large sourcedetector distance allows to recover more accurately the internal optical properties from time-resolved measurements. However, the errors in the estimated absorption coefficients $\mu_{a}$ based on the use of the time-resolved spectroscopy seem more dependent on the apple size than those obtained with the steady-state method.

The preliminary investigation of the effects of the skin layer which surrounds the flesh provides some insight into the problem that such a layer produces in apple optical spectroscopy measurements. Interestingly, Monte Carlo simulations show that the skin does not significantly impact the time-dependent measurements when a sufficient source-detector distance is fixed at $15 \mathrm{~mm}$. With this suitable source-detector tissue arrangement, it was also shown that photons involved in measurements probe the flesh at depths of $10-15 \mathrm{~mm}$ beneath the skin. This confirms that measurements on intact apple with time-resolved spectroscopy are more affected by the curved boundary than by the presence of the skin layer. The errors in the retrieved $\mu_{a}$ and $\mu_{s}^{\prime}$ values of the flesh are also dependent on the true optical parameter sets which serve as input data of the Monte Carlo simulations. This suggests that the errors in the estimates are lesser for certain apple varieties than for others.

The retrieved internal optical parameters of an apple interrogated with the steady-state reflectance spectroscopy seem few influenced by the effect of the skin layer. This may be attributed to what the shape of the reflectance curves generated with and without skin remains practically identical, except for measurements close to the source. However, only one optical parameter set of the skin (adapted from the literature) has been used to find these results. We expect that the range of results that can be displayed is vast, so the full influence of combined effects on light transport due to variation of optical properties of each bulk layer still awaits investigation. Furthermore, it seems interesting to draw conclusions from inspection of the same intact apple with TRS and SSM techniques.

This is the general view of the work we hope to report on at a later date.

\section{Acknowledgements}

This work was funded by the Regional Council of Pays de la Loire and supported by the AI fruit Project.

\section{References}

Alerstam, E., 2011. Optical Spectroscopy of Turbid Media: Time-Domain Measurements and Accelerated Monte Carlo Modelling. Doctoral thesis, Lund University.

Arridge, S.R., Schweiger, M., Hiraoka, M., Delpy, D.T., 1993. A finite-element approach for modeling photon transport in tissue. Med. Phys. 20, 299-309.

Aydin, E.D., Katsimichas, S., de Oliveira, C.R.E., 2005. Time-dependent diffusion and transport calculations using a finite-element-spherical harmonics method. J. Quant. Spectrosc. Radiat. Transfer 95, 39-363.

Baranyai, L., Zude, M., 2009. Analysis of laser light propagation in kiwifruit using backscattering imaging and Monte Carlo simulation. Comput. Electron. Agric. 69 (1), 33-39.

Boas, D.A., Culver, J., Stott, J., Dunn, A., 2002. Three dimensional Monte Carlo code for photon migration through complex heterogeneous media including the adult human head. Opt. Express. 10, 159-170.

Cheong, W.-F., Prahl, S., Welch, A., 1990. A review of the optical properties of biological tissues. IEEE J. Quant. Electron. 26, 2166-2185.

Contini, D., Martelli, F., Zaccanti, G., 1997. Photon migration through a turbid media slab described by a model based on diffusion approximation. Appl. Opt. 36, 4587-4599.

Cubeddu, R., D’Andrea, C., Pifferi, A., Taroni, P., Torricelli, A., Valentini, G., RuizAltisent, M., Valero, C., Ortiz, C., Dover, C., Johnson, D., 2001a. Time-resolved reflectance spectroscopy applied to the nondestructive monitoring of the internal optical properties in apples. Appl. Spectrosc. 55 (10), 1368-1374.

Cubeddu, R., D’Andrea, C., Pifferi, A., Taroni, P., Torricelli, A., Valentini, G., Dover, C., Johnson, D., Ruiz-Altisent, M., Valero, C., 2001b. Nondestructive quantification of chemical and physical properties of fruits by time-resolved reflectance spectroscopy in the wavelength range 650-1000 nm. Appl. Opt. 40, 538-543.

Deulin, X., L'Huillier, J.P., 2006. Finite element approach to photon propagation modeling in semi-infinite homogeneous and multilayered tissue structures. Eur. Phys. J. Appl. Phys. 33, 133-146.

Eccher Zerbini, P., Grassi, M., Cubeddu, R., Pifferi, A., Torricelli, A., 2002 Nondestructive detection of brown heart in pears by time resolved reflectance spectroscopy. Postharvest Biol. Technol. 25, 87-97.

Farrel, T.J., Patterson, M.S., Wilson, B.C., 1992. A diffusion theory model of spatially resolved, steady-state diffuse reflectance for the non invasive determination of tissue optical properties in vivo. Med. Phys. 19, 879-888.

Fedele, F., Eppstein, M.J., Laible, J.P., Godavarty, A., Sevick-Muraca, E.M., 2005. Fluorescence photon migration by the boundary element method. J. Comput. Phys. 210, 109-132. 
Feng, S., Zeng, F.A., Chance, B., 1995. Photon migration in the presence of a simple defect: a perturbation analysis. Appl. Opt. 34 (19), 3826-3837.

Fraser, D.G., Jordan, R.B., Künnemeyer, R., McGlone, V.A., 2003. Light distribution inside mandarin fruit during internal quality assessment by NIR spectroscopy. Postharvest Biol. Technol. 27, 185-196.

Gibson, A.P., Hebden, J.C., Arridge, S.R., 2005. Recent advances in diffuse optical imaging. Phys. Med. Biol. 50, R1-R43.

Guo, X., Wood, M.F.G., Vitkin, A., 2008. A Monte Carlo study of penetration depth and sampling volume of polarized light in turbid media. Opt. Commun. 281, 380-387.

Haskell, R.C., Svaasand, L.O., Tsay, T.T., Feng, T.C., McAdams, M., Tromberg, B.J., 1994. Boundary conditions for the diffusion equation in radiative transfer. J. Opt. Soc. Am. A 11, 2727-2741.

Homutová, I., Blažek, J., 2006. Differences in fruit skin thickness between selected apple (Malus domestica Borkh.) cultivars assessed by histological and sensory methods. Hort. Sci. 33 (3), 108-113 (Prague).

Keijzer, M., Richards-Kortrum, R., Jacques, S.L., Feld, M., 1989. Fluorescence spectroscopy of turbid media : autofluorescence of human aorta. Appl. Opt. 28, 4286-4292.

Keshavayzpour, F., Rashidi, M., 2011. Prediction of apple mass based on some geometrical properties using linear regression models. Acad. J. Plant Sci. 4 (4), 118-123.

Kienle, A., Patterson, M.S., 1996. Determination of the optical properties of turbid media from a single Monte Carlo simulation. Phys. Med. Biol. 41, 2221-2227.

Konarska, A., 2013. The structure of the fruit peel in two varieties of Malus domestica Borkh (Rosaceae) before and after storage. Protoplasma 250, 701-714.

Lu, R., 2004. Multispectral imaging for predicting firmness and soluble solids content of apple fruit. Postharvest Biol. Technol. 31 (2), 147-157.

Lu, R., Peng, Y., 2007. Development of a multispectral imaging prototype for realtime detection of apple fruit firmness. Opt. Eng. 46 (12), 123201

Lu, R., Qin, J., Peng, Y., 2006. Measurement of the optical properties of apples by hyperspectral imaging for assessing fruit quality. in: ASABE Annual International Meeting, Paper no. 066179, pp. 6941-6951.

Lu, R., Cen, H., Huang, M., Ariana, D.P., 2010. Spectral absorption and scattering properties of normal and bruised apple tissue. ASABE 53 (1), 263-269.

Mansouri, C., L'Huillier, J.P., Kashou, N., Humeau, A., 2010. Depth sensitivity analysis of functional near-infrared spectroscopy measurement using three-dimensional Monte Carlo modelling-based magnetic resonance imaging. Lasers Med. Sci. 25, 431-438.

Martelli, F., Sassaroli, A., Yamada, Y., Zaccanti, G., 2000. Method for measuring the diffusion coefficient of homogeneous and layered media. Opt. Lett. 25, $1508-1510$.

Nguyen Do Trong, N., Tsuta, M., Erkinbaeva, C., Mathijsa, F., Moredac, G., Barreiroc, P., Verbovena, P., Nicolaïa, B., Saeys, W., 2013. Nondestructive quality evaluation and monitoring of Braeburn apples by Spatially Resolved Spectroscopy. In: InsideFood Symposium, 9-12 April 2013, Leuven, Belgium.

Nguyen Do Trong, N., Erkinbaev, C., Tsuta, M., De Baerdmaeker, J., Nicolaï, B., Saeys, W., 2014. Spatially resolved diffuse reflectance in the visible and near-infrared wavelength range for non-destructive quality assessment of "Braeburn" apples. Postharvest Biol. Technol. 91, 39-48.

Nicolai, B.M., Beullens, K., Bobelyn, E., Peirs, A., Saeys, W., Theron, K., Lammertyn, J., 2007. Nondestructive measurement of fruit and vegetable quality by means of NIR spectroscopy: a review. Postharvest Biol. Technol. 46, 99-118.

Okada, E., Firbank, M., Schweiger, M., Arridge, S.R., Cope, M., Delpy, D.T., 1997. Theoretical and experimental investigation of near-infrared light propagation in a model of the adult head. Appl. Opt. 36, 21-31.

Park, B., Abbott, J.A., Lee, K.J., Choi, C.H., Choi, K.H., 2003. Near-infrared diffuse reflectance for quantitative and qualitative measurement of soluble solids and firmness of Delicious and Gala apples. Trans. Am. Soc. Agric. Eng. 46, 17211731.

Peng, Y., Lu, R., 2008. Analysis of spatially resolved hyperspectral scattering images for assessing apple fruit firmness and soluble solids content. Postharvest Biol. Technol. 48 (1), 52-62.
Pifferi, A., Taroni, P., Valentini, G., Andersson-Engels, S., 1998. Real-time method for fitting time-resolved reflectance and transmittance measurements with a Monte Carlo model. Appl. Opt. 37, 2774-2780.

Pogue, B.W., Patterson, M.S., 1994. Frequency-domain optical absorption spectroscopy of finite tissue volumes using diffusion theory. Phys. Med. Biol. 39, $1157-1180$.

Prahl, S.A., 1995. The adding-doubling method. In: Welsh, A.J., Van Gemert, M.J.C. (Eds.), Optical Thermal Response of Laser Irradiated Tissue. Plenum Press, New York, pp. 101-129.

Qin, J., Lu, R., 2008. Measurement of the optical properties of fruits and vegetables using spatially resolved hyperspectral diffuse reflectance imaging technique. Postharvest Biol. Technol. 49, 355-365.

Qin, J., Lu, R., 2009. Monte Carlo simulation for quantification of light transport features in apples. Comput. Electron. Agric. 68, 44-51.

Qin, J., Lu, R., Peng, Y., 2009. Prediction of apple internal quality using spectral absorption and scattering properties. ASABE 52 (2), 499-507.

Saeys, W., Velazco-Roa, M.A., Thennadil, S.N., Ramon, H., Nicolaï, B.M., 2008. Optical properties of apple skin and flesh in the wavelength range from 350 to 2200 nm. Appl. Opt. 47, 908-919.

Sassaroli, A., Martelli, F., Zaccanti, G., Yamada, Y., 2001. Performance of fitting procedures in curved geometry for retrieval of the optical properties of tissue from time-resolved measurements. Appl. Opt. 40, 185-197.

Taroni, P., Pifferi, A., Torricelli, A., Comelli, D., Cubeddu, R., 2003. In vivo absorption and scattering properties of biological tissues. Photochem. Photobiol. Sci. 2, $124-129$

Torricelli, A., Spinelli, L., Contini, D., Vanoli, M., Rizzolo, A., Eccher, Zerbini P., 2008. Time-resolved reflectance spectroscopy for non-destructive assessment of food quality. Sens. Instrum. Food Qual. 2, 82-89.

Tuchin, V.V., 1997. Light scattering study of tissues. Phys. Uspekhi 40 (5), 495-515.

Tuchin, V.V., 2007. Tissue Optics: Light Scattering Methods and Instruments for Medical Diagnosis, second ed. SPIE Press, Bellingham, WA, USA.

Vanoli, M., Rizzolo, A., Eccher, Zerbini, P., Spinelli, L., Torricelli, A., 2009. Nondestructive detection of internal defects in apple fruit by time-resolved reflectance spectroscopy. In: Proceedings of the International Conference Environmentally Friendly and Safe Technologies for Quality of Fruit and Vegetables, Universidade do Algarve, Faro, Portugal, 14-16 January 2009.

Vanoli, M., Rizzoloa, A, Zanellac, A, Grassia, M., Spinellid, L., Cubeddu, R., Torricelli, A., 2013. Apple texture in relation to optical, physical and sensory properties. In: InsideFood Symposium, 9-12 April 2013, Leuven, Belgium.

Vaudelle, F., L'Huillier, J.P., 2013. Time-resolved optical fluorescence spectroscopy of heterogeneous turbid media with special emphasis on brain tissue structures including diseased regions: a sensitivity analysis. Opt. Commun. 304, 161-168.

Verboven, P., Nemeth, A., Abera, M.K., Bongaers, E., Daelemans, D., Estrade, P., Herremans, E., Hertog, M., Saeys, W., Vanstreels, E., Verlinden, B., Leitner, M., Nicolaï, B., 2013. Optical coherence tomography visualizes microstructure of apple peel. Postharvest Biol. Technol. 78, 123-132.

Verkruysse, W., Pickering, J.W., Beek, J.F., Keijzer, M., van Gemert, M.J.C., 1993. Modelling the effect of wavelength on pulsed dye laser treatment of port wine stains. Appl. Opt. 32, 393-398.

Wang, W., Li, C., 2013. Measurement of the light absorption and scattering properties of onion skin and flesh at $633 \mathrm{~nm}$. Postharvest Biol. Technol. 86, 494501

Wang, L.H., Jacques, S.L., Zheng, L.Q., 1995. MCML-Monte-Carlo modeling of light transport in multilayered tissues. Comput. Methods Prog. Biol. 47, 131-146.

Welch, A.J., van Gemert, M.J.C., 1995. Optical-Thermal Response of Laser-Irradiated Tissue. Springer.

Wilson, B.C., Jacques, S.L., 1990. Optical reflectance and transmittance of tissues: principles and applications. IEEE J. Quant. Electron. 26, 2186-2199.

Zamorskyi, V., 2007. The role of the anatomical structure of apple fruits as fresh cut produce. ISHS Acta Hortic. 746, 509-512.

Zolek, N.S., Liebert, A., Maniewski, R., 2006. Optimization of the Monte Carlo code for modeling of photon migration in tissue. Comput. Methods Prog. Biomed. 84, $50-57$.

Zude, M., 2008. Optical Monitoring of Fresh and Por Processed Agricultural Crops. CRC Press, Boca Raton, FL, USA. 